\title{
Slow Open Dialectical Behavior Group Therapy for Adolescents and Parents: Longitudinal Study of Unconscious Orientation
}

\author{
Aranzazu Fernandez-Rivas, 1 , Eva Sesma-Pardo,,2, Iñaki Kerexeta, ${ }^{1,2}$, Aida Diaz-Cosgaya1, \\ Esther Vivanco ${ }^{1}$, Federico Carminati ${ }^{3,4}$, Miguel Angel Gonzalez Torres ${ }^{1,2}$, Claire Fouassier ${ }^{3,4}$, \\ François Martin ${ }^{5}$, Jacques Demongeot ${ }^{6}$, Giuliana Galli Carminati ${ }^{3,4,7}$ \\ ${ }^{1}$ Psychiatry Service, Basurto University Hospital-Osakidetza, Bilbao, Spain \\ ${ }^{2}$ Neurosciences Department, Basque Country University, Bilbao, Spain \\ ${ }^{3}$ ASsociation Pour le TRAvail Groupal Thérapeutique et Social (ASTRAG) and Simposietto, Geneva, Switzerland \\ ${ }^{4}$ Société Internationale de Psychanalyse Multidisciplinaire (SIPsyM), Geneva, Switzerland \\ ${ }^{5}$ Honorary Research Fellow at CNRS, Paris, France \\ ${ }^{6}$ AGEIS, EA 7407, Faculté de Médecine, Université Grenoble Alpes, La Tronche, Grenoble, France \\ ${ }^{7}$ SNUBH (Seoul National University, Bundang Hospital) eoul National University (Bundang Hospital), Seoul, Republic of Korea \\ Email: giuliana.gallicarminati@gmail.com
}

How to cite this paper: Fernandez-Rivas, A., Sesma-Pardo, E., Kerexeta, I., DiazCosgaya, A., Vivanco, E., Carminati, F., Torres, M. A. G., Fouassier, C., Martin, F., Demongeot, J., \& Carminati, G. G. (2021). Slow Open Dialectical Behavior Group Therapy for Adolescents and Parents: Longitudinal Study of Unconscious Orientation. Psychology, 12, 756-784.

https://doi.org/10.4236/psych.2021.125047

Received: April 25, 2021

Accepted: May 24, 2021

Published: May 27, 2021

Copyright (c) 2021 by author(s) and Scientific Research Publishing Inc. This work is licensed under the Creative Commons Attribution International License (CC BY 4.0).

http://creativecommons.org/licenses/by/4.0/

(c) (i) Open Access

\begin{abstract}
Introduction: The study's objective is to explore the existence and the evolution of common behavior of the participants to four slow open groups taking part in a Dialectical Behavior Therapy (DBT) skills training. Two groups were composed of adolescents and two of their respective parents. We analyzed their evolution via the answers to the "absurd" questionnaire. Methods: The "absurd questionnaire," composed of 50 pairs of images, was administered to the participants who had to choose one image from each pair. In this experiment, we were able to submit a version of the questionnaire to the participants before forming the groups. We have analyzed their initial picture choices and how these evolved, considering the changes in the choices, the differences in the four groups, the flux, and the answers' focus. Results: In the four groups, we found statistical evidence that both the pictures' initial choices and their evolution during the training are not simply governed by randomness. The initial picture choice in each pair is highly skewed toward one of the two pictures in each pair. We compare the longitudinal evolution of the picture's choice in the four groups. Conclusions: The results show that the answers to the questions are strongly polarized already before the groups convene, revealing an initial socio-cultural bias. The group environment causes relaxation of this strong initial bias and subsequent recovery. This relaxation could indicate the formation of a "group continuum," coming from the entangle-
\end{abstract}


ment of individuals' psyches, creating a group entity having its own identity. In the slow-open groups, the participants are mainly subject to clan loyalties.

\section{Keywords}

Dialectical Behavior Therapy (DBT), Group Dynamics in Young and Parents, Slow Open Group Work, Group Analysis, Unconscious Orientation

\section{Introduction}

This work is the continuation of our investigation in the possibility of quantifying or, at the least, objectifying group dynamics (Fernandez-Rivas et al., 2020; Trojaola-Zapirain et al., 2014, 2015, 2016, 2019). We have already described in the referenced works the basic principles that have led us to initiate this work, and therefore we will give here only a short reminder. The study's objective is to explore the existence and the evolution of common behavior of the participants to four slow open groups taking part in a Dialectical Behavior Therapy (DBT) skills training. Two groups were composed of adolescents and two of their respective parents. We analyzed their evolution via the answers to the "absurd" questionnaire.

Dialectical Behavioral Therapy (DBT) (Linehan, 1993, 2015; Miller et al., 2017) has demonstrated an effective transdiagnostic treatment for adolescents whose main symptoms are emotional dysregulation and impulsivity. A complete program based on this therapy has been implemented in the Psychiatric Service of Basurto University Hospital (Bilbao, Spain). This therapeutic system involves several modules, including DBT skills groups for adolescents and parents.

According to Bion, there are universal principles-that he calls "basic assumptions"-guiding the behavior of the group and its members in continual interaction with the external and internal realities of the group itself (Bion, 1961; Foulkes, 1964; Vergopoulos, 1983). This theory has led some psychoanalysts to suppose a "group" nature of the individual psyche (Kaës, 2010).

Bion also formulated the hypothesis of the existence of a group psychical apparatus. This hypothesis means that when the "basic assumptions" govern the group's behavior, the group members cannot be considered as separated entities but rather as the expression of a single psychical entity (Bion, 1961). It is intriguing to observe that this occurs for interacting quantum objects forming an entangled state (Aspect et al., 1982; Bell, 1964, 1966; Bohr, 1935; Einstein et al., 1935; Richens et al., 2017; Schrödinger, 1935, 1936).

Although never really interested in the study of the "collective," from which, according to him, the individual has to emerge via the process of individuation, Carl Gustav Jung has nevertheless provided one of the most potent metaphors for the "collective soul". According to him (Jung \& McGuire, 1925), the individual psyche lies upon a stratified unconscious in which each successive layer be- 
longs to a broader community of people and even, for the most profound strata, living beings. This "collective unconscious" (Jung, 1959) is the "locus" of the connections between the world of reality and the world of the soul. These connections appear "acausal" but, according to Jung (Jung, 1952, 1960), respond to the unfathomable logic of the unknowable collective unconscious.

The emergence of these connections into the sensible world gives rise to what Jung termed "synchronicity" (Jung, 1952), the meaningful correlation between an inner condition-feeling or thought-and an external event with no apparent causal connection.

When Jung met W. Pauli, one of the most profound and prolific physicists of the $20^{\text {th }}$ century, the concept of synchronicity was connected to the newly discovered phenomenon of quantum entanglement that will become a fundamental-albeit controversial-cornerstone of the new physics of quantum phenomena. This gave rise to a life-long intellectual collaboration between Jung and Pauli in pursuit of the dream to root both the description of the material world and that of the soul in the same basic principles (Jung et al., 2001).

Humbly following in their footsteps, we have described the interaction between the unconscious in terms of quantum entanglement. This description supposes the existence of a universal quantum field of (un)consciousness (Baaquie \& Martin, 2005; Orlov, 1982) relating all living creatures. Therefore, two interacting individuals would temporarily lose their individuality and form a connected system during their interaction. (Galli Carminati et al., 2017; Galli Carminati \& Martin, 2008; Martin et al., 2009, 2010, 2013). It is natural to extend such a model to a group of individuals (Grinberg-Zylberbaum et al., 1994; Martin \& Galli Carminati, 2009), where the entanglement between the different unconscious can bring to the formation of a single entity with a distinct behavior, explaining the correlations observed between group members (Marshall, 1989). These developments are part of a larger discipline known as psychophysics (Beck \& Eccles, 1992; Conte et al., 2003; Freeman \& Vitiello, 2016; Hameroff \& Penrose, 1996; Penrose, 1989, 1994; Pitkänen, 1998, 2010; Sabbadini \& Vitiello, 2019; Vitiello, 2003; Zurek, 1981).

In a recent series of works (Galli Carminati \& Carminati 2006; Galli Carminati \& Martin, 2008; Martin, Carminati, \& Galli Carminati, 2009; Martin, Carminati, \& Galli Carminati, 2010; Martin, Carminati, \& Galli Carminati, 2013), some of the authors of the present paper have focused on the possibility to describe the group psyche with concepts and models borrowed from Quantum Mechanics.

Some of the authors of the present work have formulated the hypothesis that, following the basic tenets of psychophysics, also the group psyche could be described by a multi-body entangled system (Galli Carminati \& Martin, 2008; Martin et al., 2009, 2010, 2013; Martin \& Galli Carminati, 2009). This hypothesis has led to the formulation of the "absurd experiment" that is the subject of this paper, and that has already been performed on different psychodynamics groups (Fernandez-Rivas et al., 2020; Trojaola-Zapirain et al., 2014, 2015, 2016, 2019). 
Although the concept of entanglement of the unconscious is not new (Atmanspacher et al., 2002; Atmanspacher \& Fach, 2013; Fach, 2011; Khrennikov, 2015; Walach \& Römer, 2011), we failed to find in the literature an experiment similar to the one presented in this paper, which seems to be therefore entirely original.

The rationale of conducting this experiment is to try to quantify, or at least objectify, one aspect of the unconscious, measuring its effect on the behavior of the participants in this study. The authors have made the hypothesis that the group environment could "amplify" a "microscopic" entanglement between the unconscious to render it detectable at the macroscopic level. The present experiment is similar to the previous ones conducted by the same authors on training groups (Trojaola-Zapirain et al., 2014, 2015, 2016, 2019) and on DBT closed groups (Fernandez-Rivas et al., 2020). In this latter study, we have introduced a "questionnaire zero" to evaluate the socio-cultural bias in the choices before the groups meet. The novelty of the present study is that it uses the same experimental setup on two "slow open" groups of adolescents and their parents who were following a Dialectical Behavior Therapy (DBT) skills training at the Psychiatric Service of Basurto University in Bilbao, Spain.

This is a setup different from the ones considered before since it cannot be considered any longer a "closed" system, but rather two couples of systems interacting with each other and also, necessarily, open to the environmental and existential situations and influences of the larger social group.

The perspective of this study is to observe the evolution of the answers to the questionnaire, considering whether the group dynamics is also tributary of the larger social group interacting as the environment in quantum mechanics.

\section{Materials and Methods}

\subsection{Participants}

Four different groups of participants are included in this study: two composed of adolescents participating in Dialectical Behavior Therapy (DBT) skills training and two others consisting of their respective parents, also participating in DBT skills training. The demography of the groups is presented in Table 1.

The adolescents followed two slow-open DBT skills training groups of 2-hour sessions on a weekly basis. One of the participants was excluded from the study because she did not participate in any of the group sessions. The group of parents was a slow open group of 10 DBT sessions of 1.5 hours each, also on a weekly basis.

The study was made after the approval of the Basurto University Hospital Ethics Committee (Bilbao, Spain) in adherence to the Helsinki Declaration for research with human subjects. All participants gave written informed consent after receiving oral and written information about the experiment, and specifically for adolescents, both the participant and their parents or legal tutor signed informed consents. All participant data were coded so that they were completely anonymous, 
Table 1. Demographic, social, and group composition of the participant sample with the age of participants in each group, adolescent and member of their family.

\begin{tabular}{ccccc}
\hline & Adolescent G1 & Adolescent G2 & Parents G1 & Parents G2 \\
\hline Total & 21 & 16 & 10 & 34 \\
Female & $14(66.7 \%)$ & $14(87.5 \%)$ & $5(50 \%)$ & $24(70.6 \%)$ \\
Average age & 15.6 & 16.4 & & \\
1Q-3Q & $14.9-16.5$ & $15.3-17.4$ & \\
Biological Family & 8 & 7 & \\
Adoptive Family & 1 & 2 & \\
Single Parent & 10 & 6 & \\
Other living situations & 2 & 1 & \\
Undergrad education & 17 & 9 & \\
Graduate education & 4 & 6 & \\
Postgraduate education & & 1 & \\
\hline
\end{tabular}

including for the researchers analyzing the data.

\subsection{Procedure}

The skills taught in these groups are mindfulness, distress tolerance, regulation of emotions, interpersonal effectiveness, and walking the middle path. The group for adolescents consists of 162 -hour sessions on a weekly basis. And the group for parents consists of 10 sessions of 1.5 hours duration, also on a weekly basis.

The groups included in this study were "slow-open," which means that participants could enter and leave the group during the training. Each group was run by two therapists.

Prior to joining the group, all participants (adolescents and parents) attended an interview for evaluation and information on the methodology of the group and the research. During that interview, participants filled the informed consent and the questionnaire number "zero" (with one exception). In addition, sociodemographic data on adolescents were collected (see Table 1).

Each participant was given an identification code to keep his identity anonymous for the research. The general setting of this experiment has been extensively described in previous publications (Fernandez-Rivas et al., 2020; Trojaola-Zapirain et al., 2014, 2015, 2016, 2019). For the purpose of this work, it will be enough to recall that we have used a questionnaire composed of 50 pairs of figures. Participants were asked to select one picture from each pair and to complete the questionnaire in three minutes. The pictures' choice aims to minimize the socio-cultural bias introduced by a word questionnaire (Zanello et al., 2004). The figures in each pair were always the same, but the pairs were randomly reshuffled at each repetition of the test to minimize mnemonic effects. Figure 1 reports a sample page from the questionnaire with fictional picture choices. 

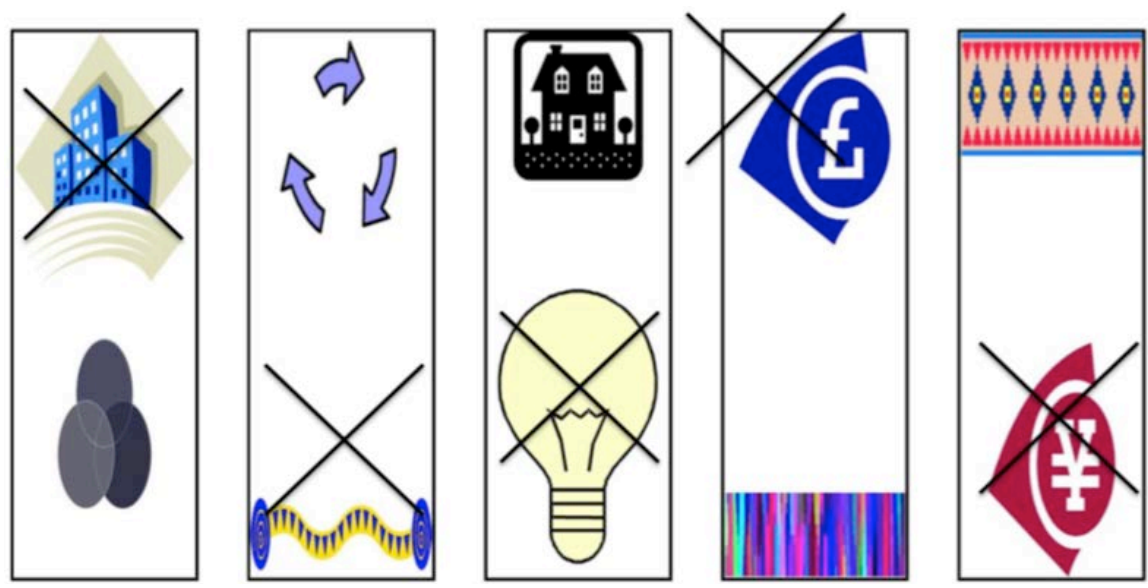

Figure 1. A page from the questionnaire with "fake" answers.

We will name the four groups PG1 (first parent group), PG2 (second parent group), YG1 (first adolescent group), and YG2 (second adolescent group). Not all the parents participated in the groups. The duration of the four training courses is, respectively, $15,55,43$, and 31 sessions.

The participation has been somewhat uneven to the different groups (see Figures 2-4). The presence in the group of adolescents was less regular after the $5^{\text {th }}$ session.

\subsection{Data Analysis}

The most frequently chosen picture in each pair during the "zero" test (the questionnaire administered before the first meeting) is indicated as picture $\mathrm{A}\left(\mathrm{A}_{i}, i=\right.$ $1,50)$, while the other picture is designed as $B\left(B_{i}, i=1,50\right)$. Frequency tables are computed for each pair of images and each session for the four groups. Because the present work is focused on the influence of the group unconscious on the measured effects-i.e., the answers to the questionnaire-we consider only the proportion of the participants choosing picture A or B for each of the 50 questions, irrespectively of how the individual participant's choice evolved.

Given the very uneven participation, we decided not to correct for data that were completely missing. Some of the sheets were incorrectly marked, and those we corrected with an LOCF (Last Observation Carry Forward, (Hamer \& Simpson, 2009)) algorithm, using the same answer from the previous session. If this was not possible, the session before was used and so on. There was no instance of invalid questionnaires for both the zero and first sessions. The total number of corrections is reported in Table 2.

\section{Results}

\subsection{Evolution of the Most Chosen Picture}

We report the evolution of the A's (most chosen picture in the questionnaire zero) for the four groups during the training. We have compared the percentage of the choice of the " $\mathrm{A}$ " picture for the 50 questions in the successive sessions. 


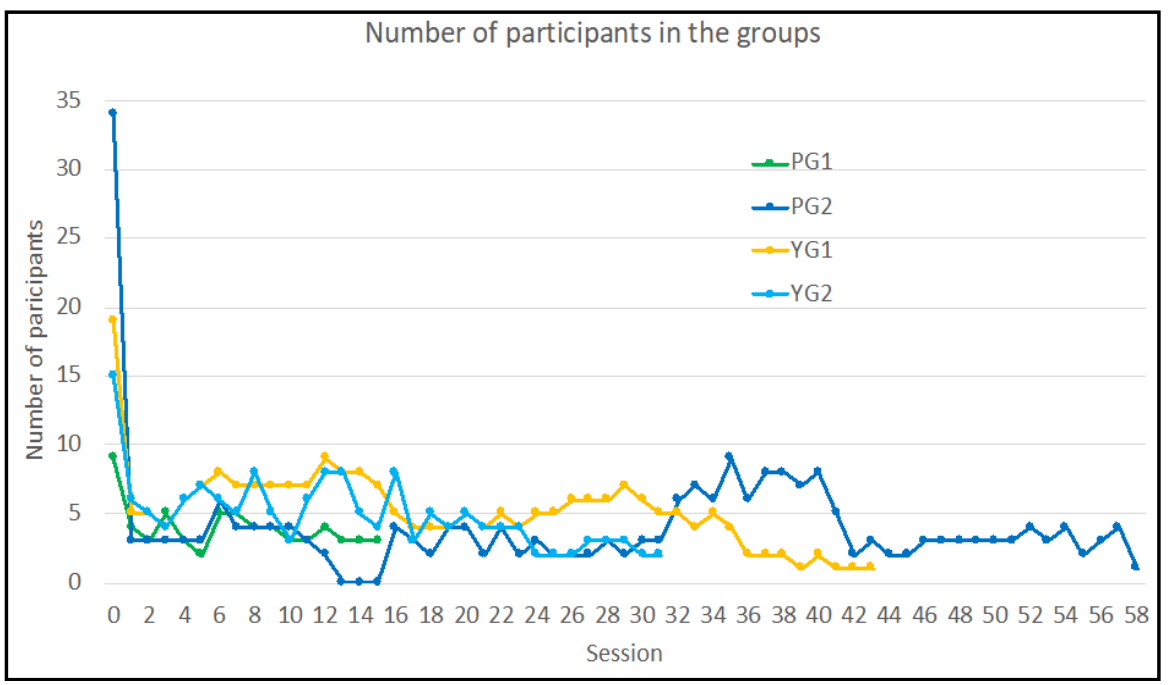

Figure 2. Number of participants in the different sessions. Note that the session number runs to 58 for the PG1 group, but three sessions were empty, and we removed them from the data.

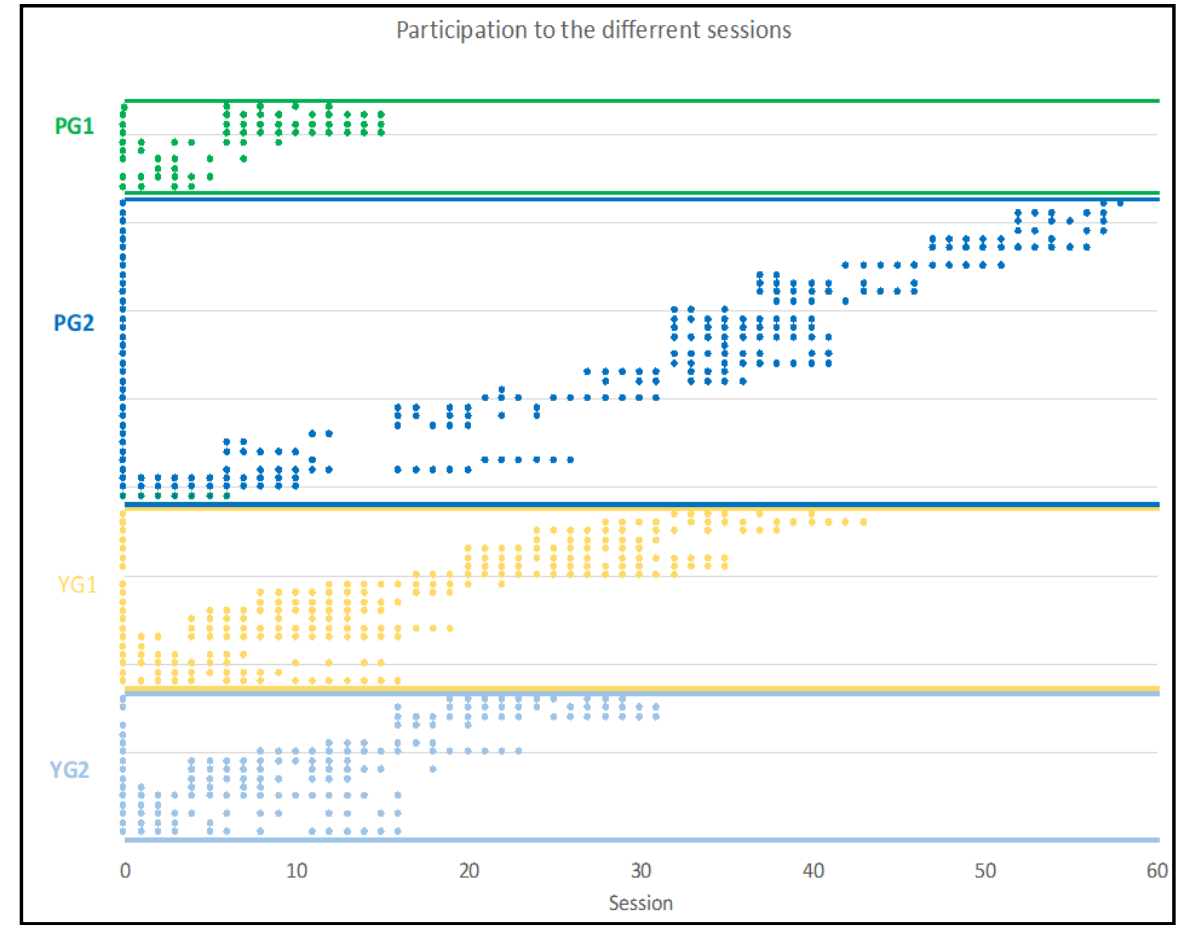

Figure 3. Participation of the individual trainees at the different sessions.

For this, we have performed a Friedmann test on the whole set of the <number of sessions $>\times 50$ data, and then, if significant-which has always been the casewe have performed a post-hoc Conover test (Conover, 1999; Conover \& Iman, 1979) between successive sessions. All the data analysis has been done with the $R$ software (R Core Team, 2020).

We show the statistically significant difference in the choice of A's between sessions, including the initial questionnaire (see Figure 5 and Table 3). 


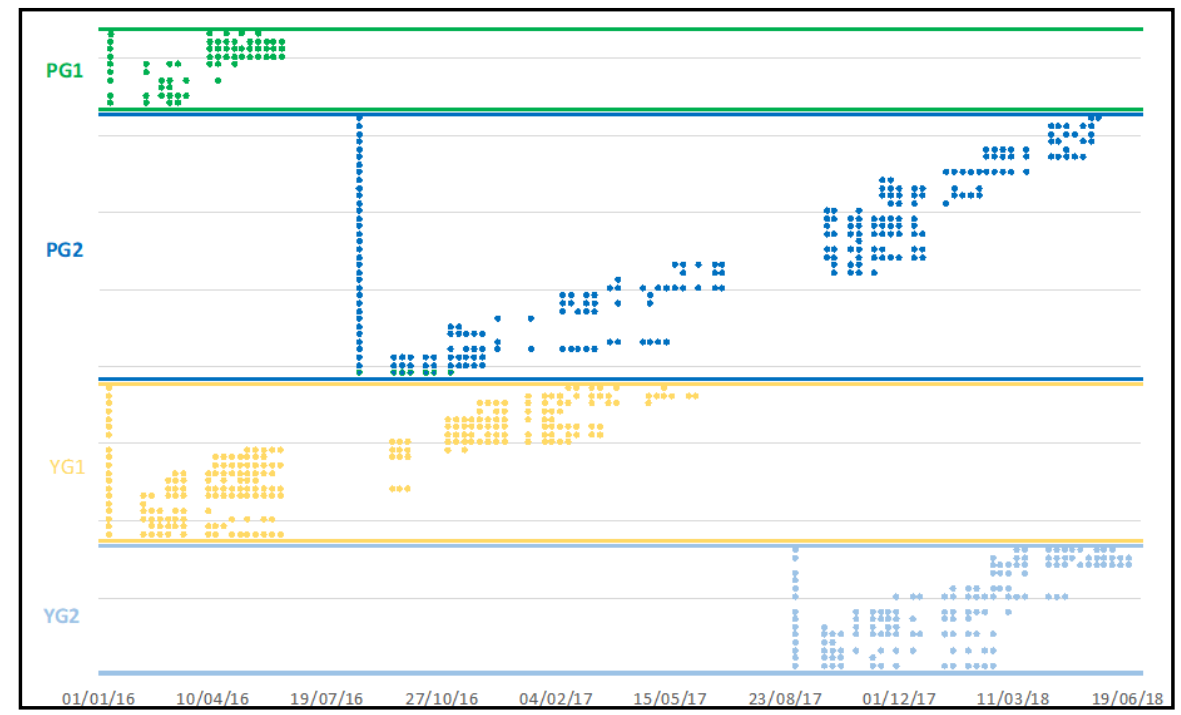

Figure 4. Evolution of the presence to the training versus time.

Table 2. Corrections to the data with LOCF.

\begin{tabular}{cccc}
\hline Group & Total valid answers & Answers corrected with LOCF & $\%$ \\
\hline PG1 & 2688 & 11 & $0.4 \%$ \\
PG2 & 10,146 & 104 & $1.0 \%$ \\
YG1 & 11,131 & 69 & $0.6 \%$ \\
YG2 & 7037 & 63 & 0.9 \\
\hline
\end{tabular}

\subsection{Evolution of the Transitions between Questionnaires}

We consider now how the choice of A and B has evolved comparing the changes of choice of the participants. For each couple of successive sessions, we count the number of participants whose choice has changed from A to B and the number of those whose choice has changed from $B$ to A. We indicate this transition with the number of the second session, i.e., we indicate with $n$ the transition $(\mathrm{A} \rightarrow \mathrm{B}$ or $\mathrm{B} \rightarrow \mathrm{A}$ ) between session $n-1$ and session $n$. To avoid repetitions, we will refer to this explanation as the notation explained in the section "Evolution of the transition between questionnaires."

We first consider the number of changes from $\mathrm{A} \rightarrow \mathrm{B}$. The statistical differences we found for the four groups are reported in Table 4 and Figure 6.

We consider now the same statistics but for the transitions B $\rightarrow$ A for the four groups. The results are shown in Figure 7 and Table 5.

We now investigate whether the individual changes of choice $\mathrm{A} \rightarrow \mathrm{B}$ and $\mathrm{B} \rightarrow$ $A$ are simple statistical fluctuations in people's preference or whether there is a statistically significant difference. This may indicate that the change of choice of the group taken as an ensemble is not casual and that there is a significant movement either toward the initial choice or away from it. As before, we indicate with $n$ the change from $\mathrm{A} \rightarrow \mathrm{B}$ or $\mathrm{B} \rightarrow \mathrm{A}$ between session $n-1$ and session $n$. We will call this the non-casuality plot. 
Table 3. Comparison of the choices of the "A picture" between successive sessions with the Conover post-hoc test. Two sessions are statistically different when $\mathrm{p}<0.05$, and the corresponding cell is red in the table.

\begin{tabular}{|c|c|c|c|c|c|c|c|c|c|}
\hline Comparison & PG1 & PG2 & YG1 & YG2 & Comparison & PG1 & PG2 & YG1 & YG2 \\
\hline $\mathrm{A} 00-\mathrm{A} 01$ & 0.95 & 0.87 & 0.43 & 0.02 & A27-A28 & & 0.35 & 0.36 & 0.01 \\
\hline A01-A02 & 0.87 & 0.35 & 0.53 & 0.15 & A28-A29 & & 0.05 & 0.49 & 0.02 \\
\hline A02-A03 & 1.00 & 0.20 & 0.98 & 0.06 & A29-A30 & & 0.70 & 0.05 & 0.04 \\
\hline A03-A04 & 0.00 & 0.87 & 0.35 & 0.00 & A30-A31 & & 0.86 & 0.69 & 0.38 \\
\hline A04-A05 & 0.00 & 0.74 & 0.91 & 0.98 & A31-A32 & & 0.53 & 0.78 & \\
\hline A05-A06 & 0.03 & 0.67 & 0.61 & 0.13 & A32-A33 & & 0.86 & 0.39 & \\
\hline A06-A07 & 0.35 & 0.23 & 0.22 & 0.89 & A33-A34 & & 0.72 & 0.99 & \\
\hline A07-A08 & 0.70 & 0.30 & 0.93 & 0.57 & A34-A35 & & 0.88 & 0.51 & \\
\hline A08-A09 & 0.58 & 0.42 & 0.18 & 0.25 & A35-A36 & & 0.81 & 0.92 & \\
\hline A09-A10 & 0.00 & 0.42 & 0.90 & 0.08 & A36-A37 & & 0.93 & 0.46 & \\
\hline A10-A11 & 0.00 & 0.07 & 0.67 & 0.00 & A37-A38 & & 0.18 & 0.01 & \\
\hline A11-A12 & 0.02 & 0.58 & 0.45 & 0.88 & A38-A39 & & 0.70 & 0.19 & \\
\hline A12-A13 & 0.20 & 0.45 & 0.19 & 1.00 & A39-A40 & & 0.11 & 0.12 & \\
\hline A13-A14 & 0.92 & 0.91 & 0.38 & 0.92 & $\mathrm{~A} 40-\mathrm{A} 41$ & & 0.09 & 0.00 & \\
\hline A14-A15 & 0.78 & 0.37 & 0.67 & 0.97 & A41-A42 & & 0.33 & 0.15 & \\
\hline A15-A16 & & 0.67 & 0.44 & 0.17 & A42-A43 & & 0.15 & 0.95 & \\
\hline A16-A17 & & 0.88 & 0.06 & 0.53 & A43-A44 & & 0.49 & & \\
\hline A17-A18 & & 0.15 & 0.00 & 0.70 & A44-A45 & & 0.94 & & \\
\hline A18-A19 & & 0.41 & 0.52 & 0.48 & A45-A46 & & 0.75 & & \\
\hline A19-A20 & & 0.01 & 0.00 & 0.76 & A46-A47 & & 0.93 & & \\
\hline A20-A21 & & 0.00 & 0.44 & 0.74 & A47-A48 & & 0.83 & & \\
\hline $\mathrm{A} 21-\mathrm{A} 22$ & & 0.04 & 0.16 & 0.67 & A48-A49 & & 0.44 & & \\
\hline A22-A23 & & 0.87 & 0.25 & 0.09 & A49-A50 & & 0.66 & & \\
\hline A23-A24 & & 0.54 & 0.97 & 0.87 & A50-A51 & & 0.19 & & \\
\hline A24-A25 & & 0.53 & 0.55 & 0.31 & A51-A52 & & 0.99 & & \\
\hline A25-A26 & & 0.23 & 0.93 & 0.44 & A52-A53 & & 0.60 & & \\
\hline A26-A27 & & 0.13 & 0.68 & 0.02 & A53-A54 & & 0.03 & & \\
\hline
\end{tabular}


A. Fernandez-Rivas et al.

Table 4. Comparison of the change of choices $\mathrm{A} \rightarrow \mathrm{B}$ between successive sessions with the Conover post-hoc test. Two changes of choices are statistically different when $p<0.05$, and the corresponding cell is red in the table.

\begin{tabular}{|c|c|c|c|c|c|c|c|c|c|}
\hline Change $\mathrm{A} \rightarrow \mathrm{B}$ & PG1 & PG2 & YG1 & YG2 & Change $\mathrm{A} \rightarrow \mathrm{B}$ & PG1 & PG2 & YG1 & YG2 \\
\hline $00 \rightarrow 01-01 \rightarrow 02$ & 0.00 & 0.13 & 0.24 & 0.69 & $27 \rightarrow 28-28 \rightarrow 29$ & & 0.01 & 0.67 & 0.01 \\
\hline $01 \rightarrow 02-02 \rightarrow 03$ & 0.00 & 0.02 & 0.40 & 0.04 & $28 \rightarrow 29-29 \rightarrow 30$ & & 0.00 & 0.19 & 0.01 \\
\hline $02 \rightarrow 03-03 \rightarrow 04$ & 0.00 & 0.32 & 0.46 & 0.01 & $29 \rightarrow 30-30 \rightarrow 31$ & & 0.04 & 0.46 & 0.02 \\
\hline $03 \rightarrow 04-04 \rightarrow 05$ & 0.00 & 0.45 & 0.27 & 0.16 & $30 \rightarrow 31-31 \rightarrow 32$ & & 0.09 & 0.21 & \\
\hline $04 \rightarrow 05-05 \rightarrow 06$ & 0.56 & 0.86 & 0.31 & 0.63 & $31 \rightarrow 32-32 \rightarrow 33$ & & 0.16 & 0.16 & \\
\hline $05 \rightarrow 06-06 \rightarrow 07$ & 0.00 & 0.19 & 0.26 & 0.86 & $32 \rightarrow 33-33 \rightarrow 34$ & & 0.80 & 0.29 & \\
\hline $06 \rightarrow 07-07 \rightarrow 08$ & 0.00 & 0.11 & 0.26 & 0.23 & $33 \rightarrow 34-34 \rightarrow 35$ & & 0.56 & 0.17 & \\
\hline $07 \rightarrow 08-08 \rightarrow 09$ & 0.12 & 0.29 & 0.72 & 0.83 & $34 \rightarrow 35-35 \rightarrow 36$ & & 0.41 & 0.00 & \\
\hline $08 \rightarrow 09-09 \rightarrow 10$ & 0.00 & 0.28 & 0.77 & 0.18 & $35 \rightarrow 36-36 \rightarrow 37$ & & 0.36 & 0.36 & \\
\hline $09 \rightarrow 10-10 \rightarrow 11$ & 0.00 & 0.00 & 0.32 & 0.07 & $36 \rightarrow 37-37 \rightarrow 38$ & & 0.16 & 0.78 & \\
\hline $10 \rightarrow 11-11 \rightarrow 12$ & 0.14 & 0.02 & 0.09 & 0.53 & $37 \rightarrow 38-38 \rightarrow 39$ & & 0.00 & 0.74 & \\
\hline $11 \rightarrow 12-12 \rightarrow 13$ & 0.76 & 0.10 & 0.27 & 0.38 & $38 \rightarrow 39-39 \rightarrow 40$ & & 0.17 & 0.01 & \\
\hline $12 \rightarrow 13-13 \rightarrow 14$ & 0.78 & 0.06 & 0.52 & 0.99 & $39 \rightarrow 40-40 \rightarrow 41$ & & 0.25 & 0.02 & \\
\hline $13 \rightarrow 14-14 \rightarrow 15$ & 0.28 & 0.06 & 0.00 & 0.38 & $40 \rightarrow 41-41 \rightarrow 42$ & & 0.31 & 0.01 & \\
\hline $14 \rightarrow 15-15 \rightarrow 16$ & & 0.68 & 0.00 & 0.83 & $41 \rightarrow 42-42 \rightarrow 43$ & & 0.37 & 0.34 & \\
\hline $15 \rightarrow 16-16 \rightarrow 17$ & & 0.04 & 0.01 & 0.53 & $42 \rightarrow 43-43 \rightarrow 44$ & & 0.15 & & \\
\hline $16 \rightarrow 17-17 \rightarrow 18$ & & 0.00 & 0.00 & 0.30 & $43 \rightarrow 44-44 \rightarrow 45$ & & 0.49 & & \\
\hline $17 \rightarrow 18-18 \rightarrow 19$ & & 0.00 & 0.04 & 0.16 & $44 \rightarrow 45-45 \rightarrow 46$ & & 0.57 & & \\
\hline $18 \rightarrow 19-19 \rightarrow 20$ & & 0.01 & 0.00 & 0.00 & $45 \rightarrow 46-46 \rightarrow 47$ & & 0.74 & & \\
\hline $19 \rightarrow 20-20 \rightarrow 21$ & & 0.37 & 0.00 & 0.16 & $46 \rightarrow 47-47 \rightarrow 48$ & & 0.97 & & \\
\hline $20 \rightarrow 21-21 \rightarrow 22$ & & 0.73 & 0.56 & 0.81 & $47 \rightarrow 48-48 \rightarrow 49$ & & 0.19 & & \\
\hline $21 \rightarrow 22-22 \rightarrow 23$ & & 0.81 & 0.71 & 0.12 & $48 \rightarrow 49-49 \rightarrow 50$ & & 0.44 & & \\
\hline $22 \rightarrow 23-23 \rightarrow 24$ & & 0.01 & 0.39 & 0.18 & $49 \rightarrow 50-50 \rightarrow 51$ & & 0.25 & & \\
\hline $23 \rightarrow 24-24 \rightarrow 25$ & & 0.02 & 0.01 & 0.00 & $50 \rightarrow 51-51 \rightarrow 52$ & & 0.57 & & \\
\hline $24 \rightarrow 25-25 \rightarrow 26$ & & 0.58 & 0.43 & 0.14 & $51 \rightarrow 52-52 \rightarrow 53$ & & 0.44 & & \\
\hline $25 \rightarrow 26-26 \rightarrow 27$ & & 0.12 & 0.90 & 0.15 & $52 \rightarrow 53-53 \rightarrow 54$ & & 0.78 & & \\
\hline $26 \rightarrow 27-27 \rightarrow 28$ & & 0.04 & 0.42 & 0.00 & $53 \rightarrow 54-54 \rightarrow 55$ & & 0.12 & & \\
\hline
\end{tabular}


Table 5. Comparison of the change of choices $B \rightarrow A$ between successive sessions with the Conover post-hoc test. Two changes of choices are statistically different when $p<0.05$, and the corresponding cell is red in the table.

\begin{tabular}{|c|c|c|c|c|c|c|c|c|c|}
\hline Change $\mathrm{B} \rightarrow \mathrm{A}$ & PG1 & PG2 & YG1 & YG2 & Change $\mathrm{B} \rightarrow \mathrm{A}$ & PG1 & PG2 & YG1 & YG2 \\
\hline $00 \rightarrow 01-01 \rightarrow 02$ & 0.00 & 0.53 & 0.74 & 0.18 & $27 \rightarrow 28-28 \rightarrow 29$ & & 0.00 & 0.83 & 0.02 \\
\hline $01 \rightarrow 02-02 \rightarrow 03$ & 0.00 & 0.83 & 0.27 & 0.01 & $28 \rightarrow 29-29 \rightarrow 30$ & & 0.00 & 0.18 & 0.31 \\
\hline $02 \rightarrow 03-03 \rightarrow 04$ & 0.73 & 0.30 & 0.48 & 0.13 & $29 \rightarrow 30-30 \rightarrow 31$ & & 0.37 & 0.08 & 0.71 \\
\hline $03 \rightarrow 04-04 \rightarrow 05$ & 0.00 & 0.92 & 0.50 & 0.79 & $30 \rightarrow 31-31 \rightarrow 32$ & & 0.42 & 0.09 & \\
\hline $04 \rightarrow 05-05 \rightarrow 06$ & 1.00 & 0.45 & 0.37 & 0.20 & $31 \rightarrow 32-32 \rightarrow 33$ & & 0.25 & 0.67 & \\
\hline $05 \rightarrow 06-06 \rightarrow 07$ & 0.00 & 0.88 & 0.27 & 0.93 & $32 \rightarrow 33-33 \rightarrow 34$ & & 0.12 & 0.91 & \\
\hline $06 \rightarrow 07-07 \rightarrow 08$ & 0.22 & 0.23 & 0.34 & 0.60 & $33 \rightarrow 34-34 \rightarrow 35$ & & 0.02 & 0.83 & \\
\hline $07 \rightarrow 08-08 \rightarrow 09$ & 0.02 & 0.79 & 0.89 & 0.78 & $34 \rightarrow 35-35 \rightarrow 36$ & & 0.12 & 0.00 & \\
\hline $08 \rightarrow 09-09 \rightarrow 10$ & 0.01 & 0.33 & 0.68 & 0.41 & $35 \rightarrow 36-36 \rightarrow 37$ & & 0.52 & 0.56 & \\
\hline $09 \rightarrow 10-10 \rightarrow 11$ & 0.00 & 0.00 & 0.58 & 0.02 & $36 \rightarrow 37-37 \rightarrow 38$ & & 0.85 & 0.95 & \\
\hline $10 \rightarrow 11-11 \rightarrow 12$ & 0.00 & 0.03 & 0.01 & 0.12 & $37 \rightarrow 38-38 \rightarrow 39$ & & 0.00 & 0.03 & \\
\hline $11 \rightarrow 12-12 \rightarrow 13$ & 0.42 & 0.01 & 0.00 & 0.12 & $38 \rightarrow 39-39 \rightarrow 40$ & & 0.03 & 0.20 & \\
\hline $12 \rightarrow 13-13 \rightarrow 14$ & 0.41 & 0.00 & 0.02 & 0.24 & $39 \rightarrow 40-40 \rightarrow 41$ & & 0.81 & 0.03 & \\
\hline $13 \rightarrow 14-14 \rightarrow 15$ & 0.12 & 0.00 & 0.00 & 0.48 & $40 \rightarrow 41-41 \rightarrow 42$ & & 0.47 & 0.11 & \\
\hline $14 \rightarrow 15-15 \rightarrow 16$ & & 0.48 & 0.00 & 0.95 & $41 \rightarrow 42-42 \rightarrow 43$ & & 0.39 & 0.67 & \\
\hline $15 \rightarrow 16-16 \rightarrow 17$ & & 0.03 & 0.36 & 0.31 & $42 \rightarrow 43-43 \rightarrow 44$ & & 0.29 & & \\
\hline $16 \rightarrow 17-17 \rightarrow 18$ & & 0.00 & 0.07 & 0.11 & $43 \rightarrow 44-44 \rightarrow 45$ & & 0.10 & & \\
\hline $17 \rightarrow 18-18 \rightarrow 19$ & & 0.00 & 0.00 & 0.41 & $44 \rightarrow 45-45 \rightarrow 46$ & & 0.63 & & \\
\hline $18 \rightarrow 19-19 \rightarrow 20$ & & 0.17 & 0.06 & 0.06 & $45 \rightarrow 46-46 \rightarrow 47$ & & 0.49 & & \\
\hline $19 \rightarrow 20-20 \rightarrow 21$ & & 0.00 & 0.09 & 0.72 & $46 \rightarrow 47-47 \rightarrow 48$ & & 0.92 & & \\
\hline $20 \rightarrow 21-21 \rightarrow 22$ & & 0.61 & 0.75 & 0.88 & $47 \rightarrow 48-48 \rightarrow 49$ & & 0.00 & & \\
\hline $21 \rightarrow 22-22 \rightarrow 23$ & & 0.03 & 0.57 & 0.63 & $48 \rightarrow 49-49 \rightarrow 50$ & & 0.07 & & \\
\hline $22 \rightarrow 23-23 \rightarrow 24$ & & 0.07 & 0.27 & 0.01 & $49 \rightarrow 50-50 \rightarrow 51$ & & 0.45 & & \\
\hline $23 \rightarrow 24-24 \rightarrow 25$ & & 0.34 & 0.83 & 0.14 & $50 \rightarrow 51-51 \rightarrow 52$ & & 0.84 & & \\
\hline $24 \rightarrow 25-25 \rightarrow 26$ & & 0.02 & 0.21 & 0.31 & $51 \rightarrow 52-52 \rightarrow 53$ & & 0.26 & & \\
\hline $25 \rightarrow 26-26 \rightarrow 27$ & & 0.09 & 0.94 & 0.01 & $52 \rightarrow 53-53 \rightarrow 54$ & & 0.28 & & \\
\hline $26 \rightarrow 27-27 \rightarrow 28$ & & 0.55 & 0.70 & 0.33 & $53 \rightarrow 54-54 \rightarrow 55$ & & 0.98 & & \\
\hline
\end{tabular}



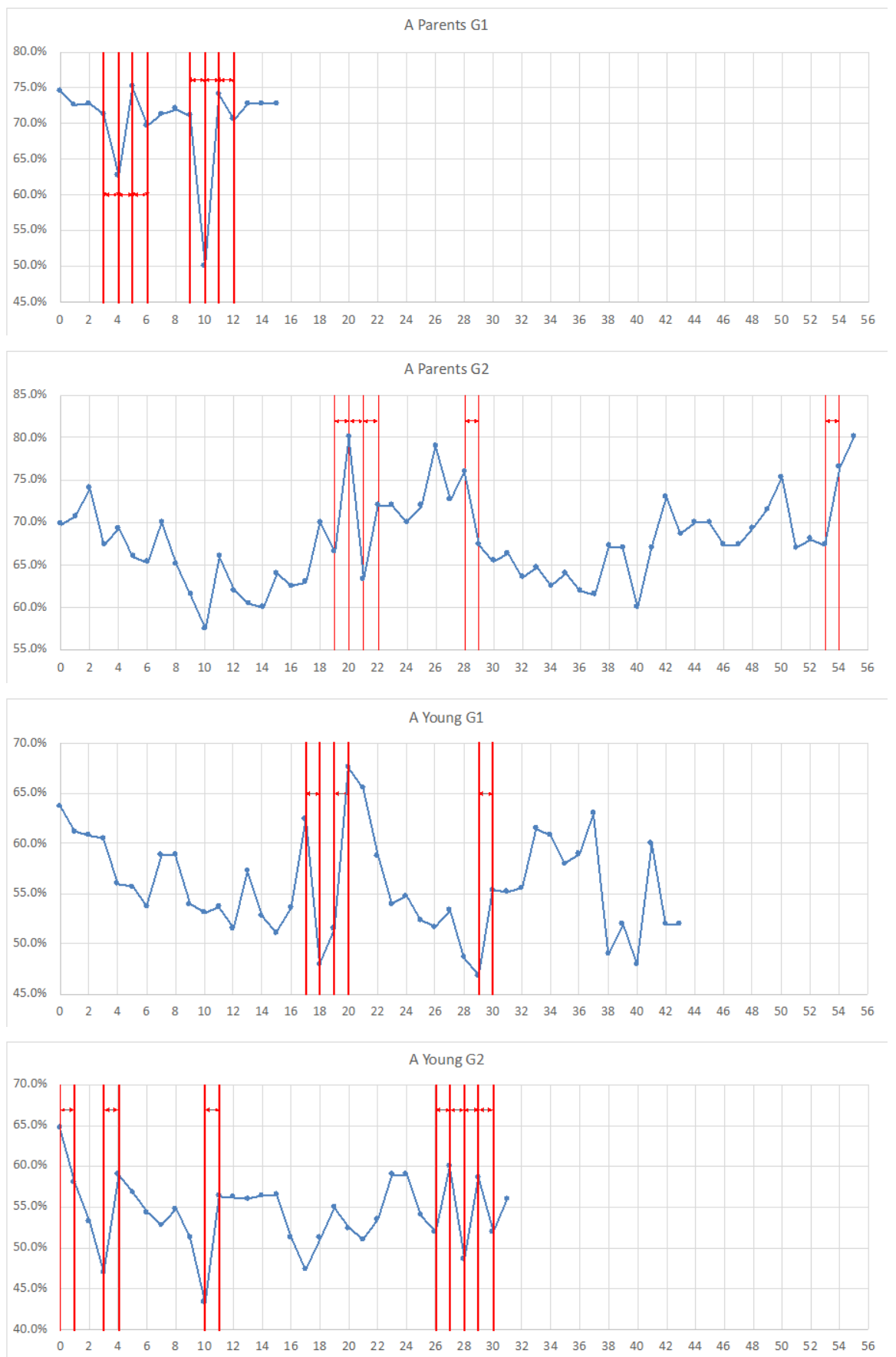

Figure 5. Evolution of the choice of A for the four groups during the training versus the session number. The arrows mark the couple of sessions for which there is a statistically significant difference $(\mathrm{p}<0.05)$ with the Conover post-hoc test.

We found statistically significant differences, considering the four groups, with the notation explained in the session "Evolution of the transitions between questionnaires," as shown in Figure 8 and Table 6.

\subsection{Evolution of "Flux" and "Focus"}

We can combine the transitions $\mathrm{A} \rightarrow \mathrm{B}$ and $\mathrm{B} \rightarrow \mathrm{A}$ into two derived quantities 


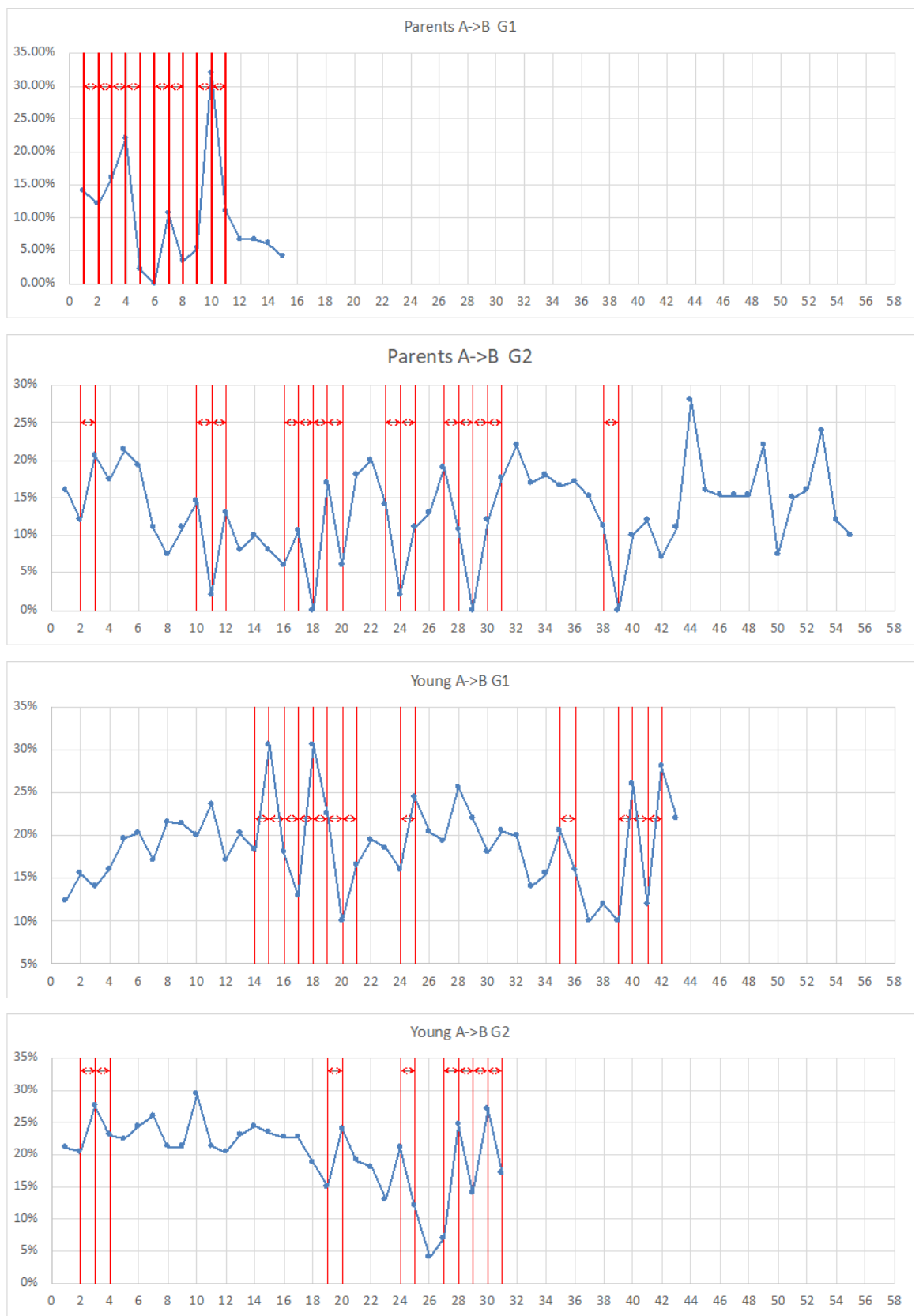

Figure 6. Evolution of the change in choice $\mathrm{A} \rightarrow \mathrm{B}$ for the four groups during the training versus the session number. The arrows mark the couple of sessions for which there is a statistically significant difference $(\mathrm{p}<0.05)$ with the Conover post-hoc test.

that help to interpret the evolution of the groups' choice of pictures. We call the quantity A $\rightarrow$ B + B $\rightarrow$ A the "flux" as it expresses the total "activity" of the group in changing the initial choice of picture. We introduce a second quantity $\mathrm{B} \rightarrow$ $\mathrm{A}-\mathrm{A} \rightarrow \mathrm{B}$ that we call "focus" as it indicates the tendency to confirm, if greater than 0 , or move away from, if smaller than 0 , the initial choice of $A$.

As in the previous section, we consider at first a Friedmann test of the whole $50 \times<$ number of sessions $>$ matrix for the focus for the four groups. All these tests indicate a significant deviation from purely random fluctuations. Following 

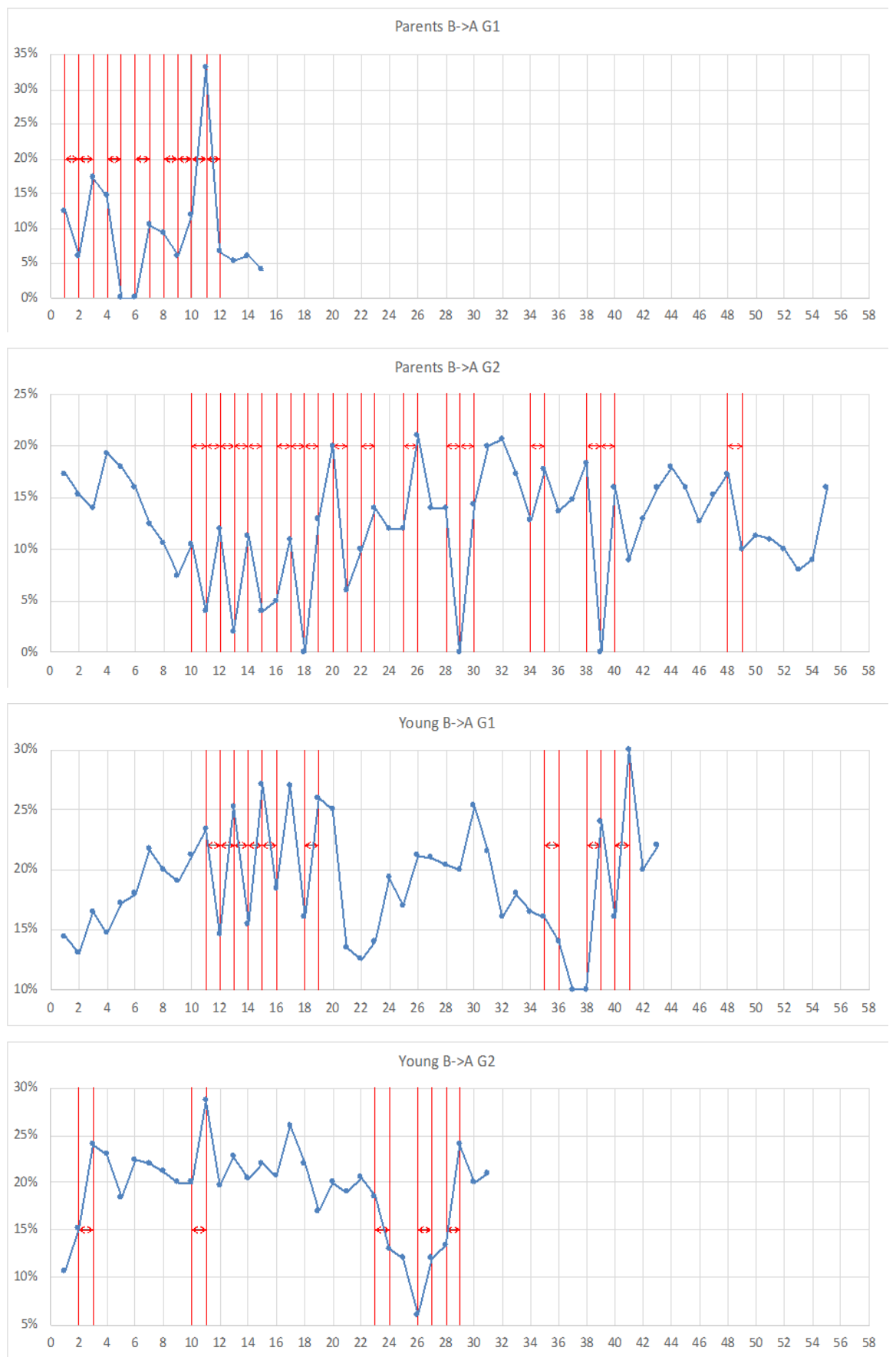

Figure 7. Evolution of the change in choice $\mathrm{B} \rightarrow \mathrm{A}$ for the four groups during the training versus the session number. The arrows mark the couple of sessions for which there is a statistically significant difference $(\mathrm{p}<0.05)$ with the Conover post-hoc test.

this, we compare each session with the following one using the Conover posthoc test for each group. The results can be seen in Figure 9 and Table 7.

We now consider the focus, i.e., the difference of the changes of choice $B \rightarrow$ $\mathrm{A}-\mathrm{A} \rightarrow \mathrm{B}$ that gives us the tendency to converge toward the initial choice-if positive-or to move away from it—if negative. The results can be seen in Figure 10 and Table 8. 

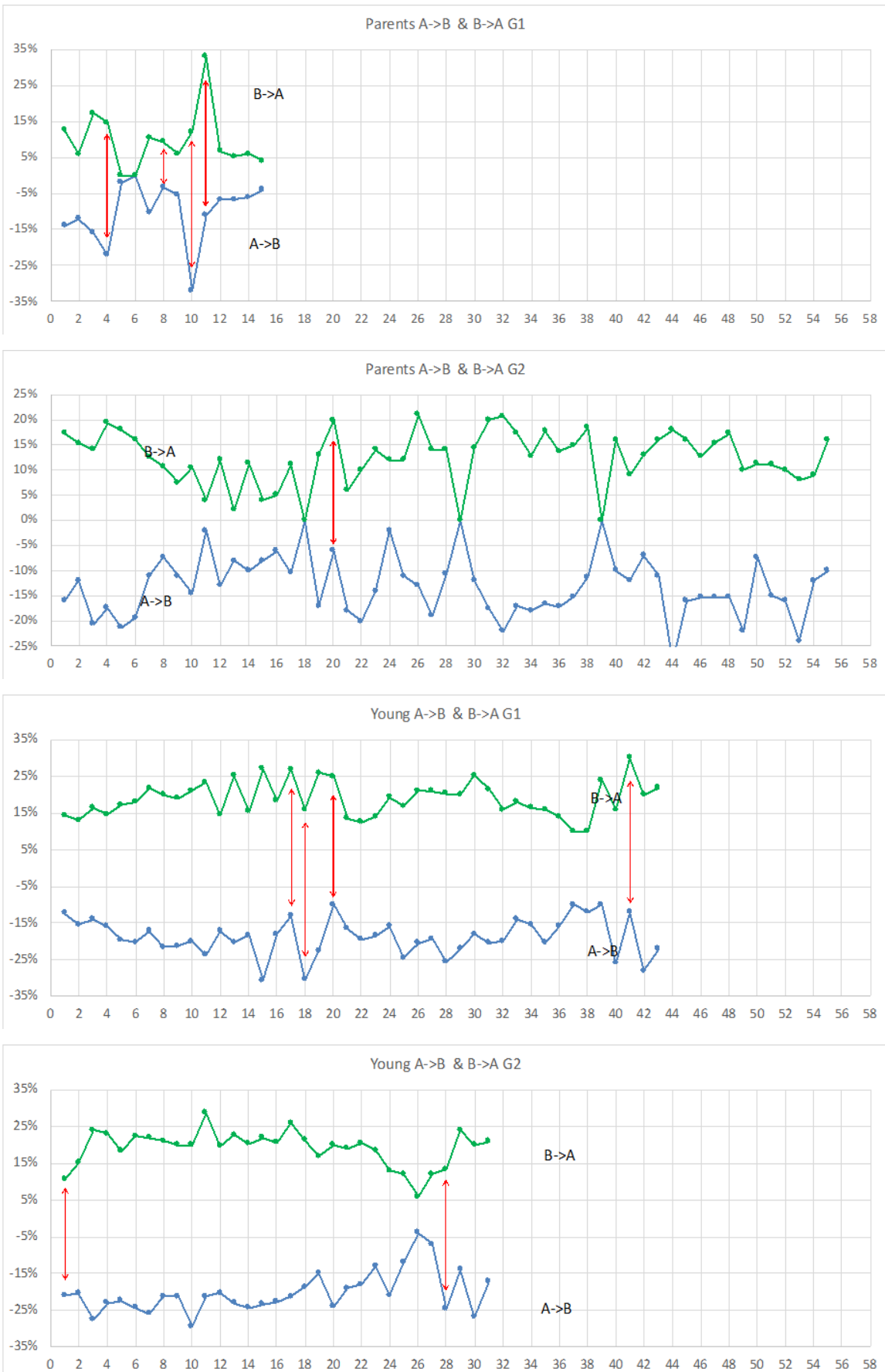

Figure 8. Comparison of the percentage of $\mathrm{A} \rightarrow \mathrm{B}$ and $\mathrm{B} \rightarrow \mathrm{A}$ changes for each group as calculated with the Conover test following a Friedmann test on the totality of the percentages. We report on the graphs the average of the percentages of changes of each type for each session, and a red arrow indicates the transitions for which there is a significant difference $(\mathrm{p}<0.05$ ) between the $\mathrm{A} \rightarrow \mathrm{B}$ and $\mathrm{B} \rightarrow \mathrm{A}$ percentages for the 50 questions. For clarity, the negative of the average of the $\mathrm{A} \rightarrow \mathrm{B}$ percentages is reported.

\subsection{Evolution of Non-Casuality, Flux, and Focus in Time}

In Figure 11, we show the evolution of the percentage of A's choice in chronological order for the four groups. 

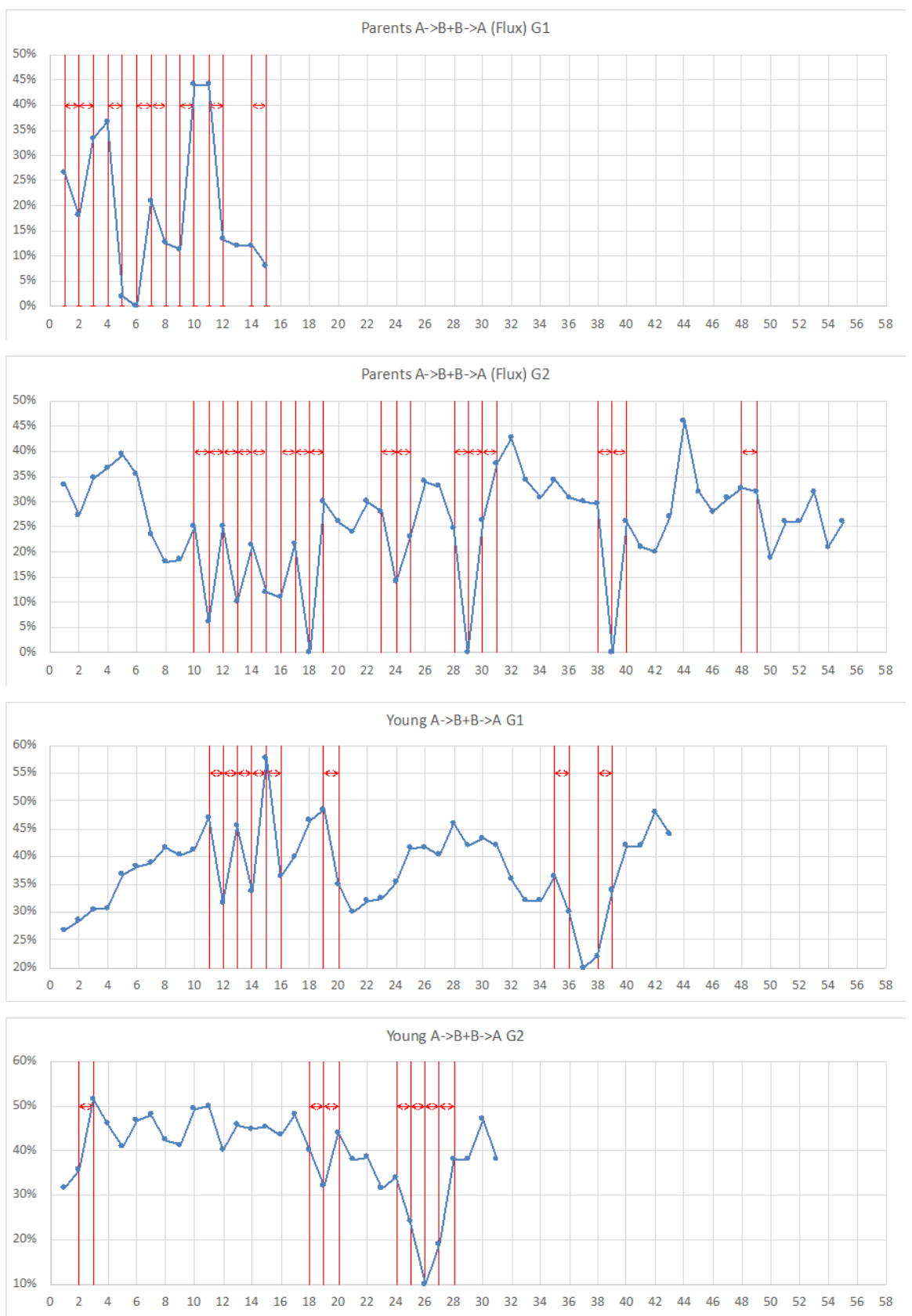

Figure 9. Comparison of the percentage of $\mathrm{A} \rightarrow \mathrm{B}+\mathrm{B} \rightarrow \mathrm{A}$ changes between successive sessions for each group as calculated with the Conover test following a Friedmann test on the totality of the percentages.

In Figure 12, we show the statistically significant differences between sessions for the percentage of A's choice and the percentage of $\mathrm{A} \rightarrow \mathrm{B}$ and $\mathrm{B} \rightarrow \mathrm{A}$ transitions in chronological order for the four groups. For the latter two, we use the notation explained in the section "Evolution of the transitions between questionnaires."

Considering the choice of $\mathrm{A}$, we show the statistically significant comparisons between consecutive sessions, including the questionnaire zero completed before the beginning of the training. For the transition $\mathrm{A} \rightarrow \mathrm{B}$ and $\mathrm{B} \rightarrow \mathrm{A}$, we use the 


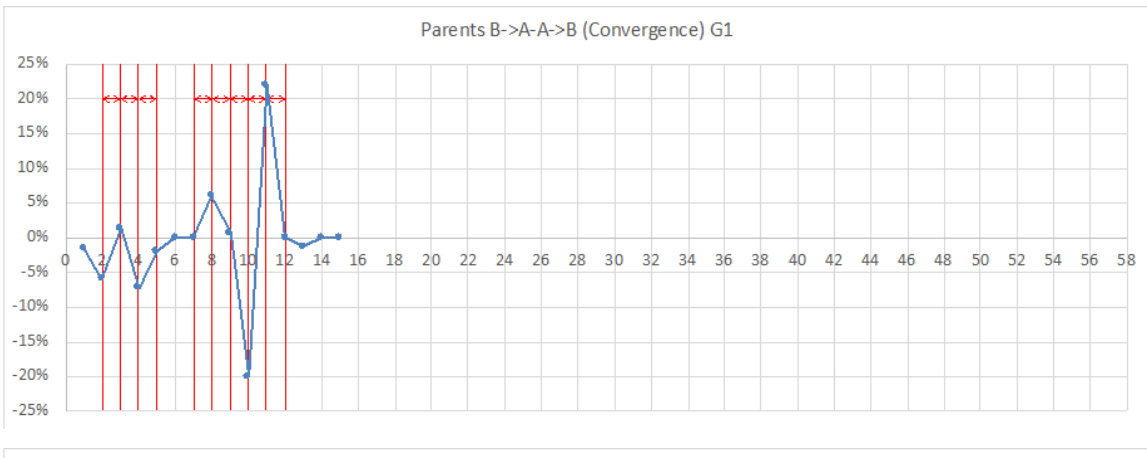

Parents B->A-A->B (Convergence) G2
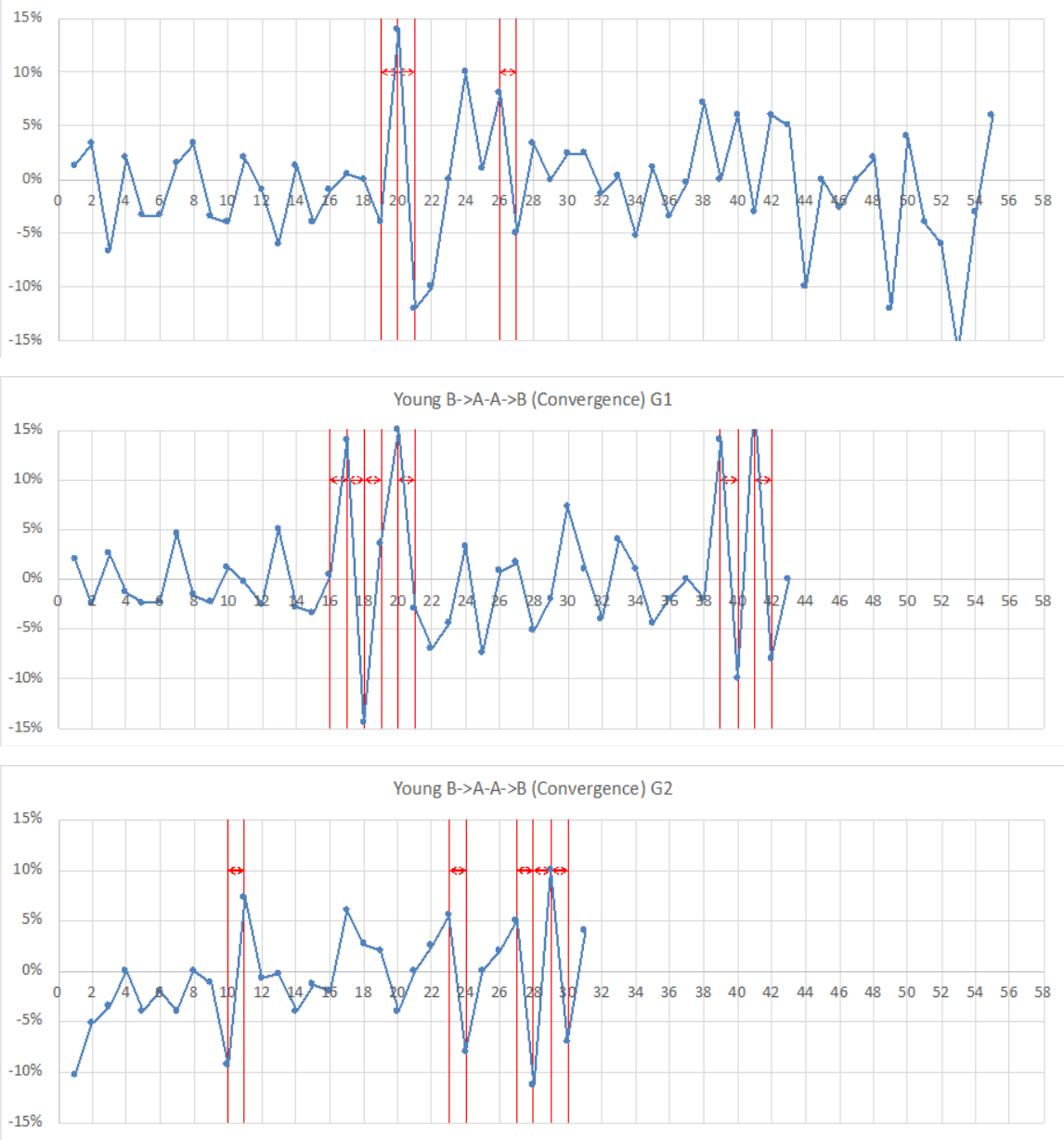

Figure 10. Comparison of the percentage of the $\mathrm{B} \rightarrow \mathrm{A}-\mathrm{A} \rightarrow \mathrm{B}$ changes between successive sessions for each group as calculated with the Conover test following a Friedmann test on the totality of the percentages.

notation explained in the section "Evolution of the transitions between questionnaires."

We note that statistically significant changes in the percentage of the A's choices happen close to the statistically significant transitions $\mathrm{A} \rightarrow \mathrm{B}$ and $\mathrm{B} \rightarrow \mathrm{A}$ for several cases in PG1, PG2, and YG2, and only twice in YG1. There are more statistically significant transitions $\mathrm{A} \rightarrow \mathrm{B}$ and $\mathrm{B} \rightarrow \mathrm{A}$ than changes in the A's choice for the four groups. 




Figure 11. Evolution of the percentage of A's choice in chronological order for the four groups.

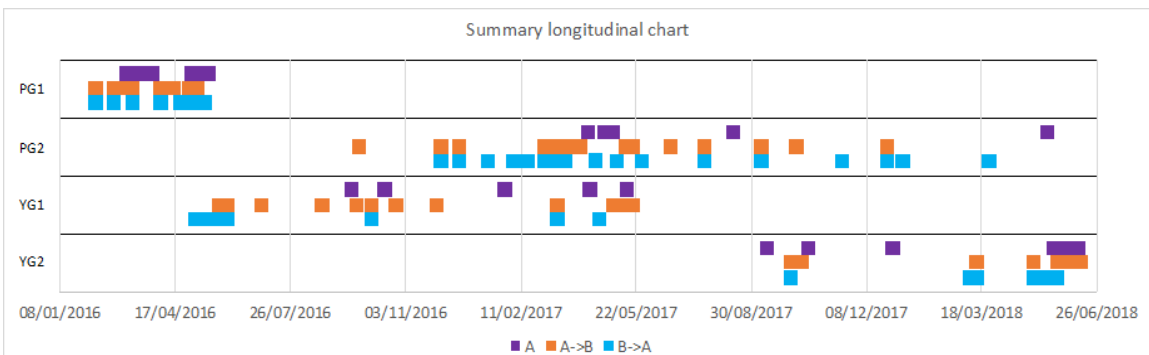

Figure 12. Statistically significant differences between sessions for the percentage of A's choice and the percentage of $\mathrm{A} \rightarrow \mathrm{B}$ and $\mathrm{B} \rightarrow \mathrm{A}$ transitions in chronological order for the four groups.

In Figure 13, we show the statistically significant differences between sessions for the percentages of focus $(\mathrm{B} \rightarrow \mathrm{A}-\mathrm{A} \rightarrow \mathrm{B})$, flux $(\mathrm{A} \rightarrow \mathrm{B}+\mathrm{B} \rightarrow \mathrm{A})$, and non-casuality ( $\rightarrow \mathrm{B}$ vs. $\mathrm{B} \rightarrow \mathrm{A}$ ) transitions in chronological order for the four groups. This allows us to determine whether there is a correlation between non-casuality, flux, and focus during the four groups' chronological evolution. We remember that we have explained the notation used for the questionnaire number in the section "Evolution of the transitions between questionnaires."

We note that the statistically significant values for non-casuality are near to statistically significant values of flux and focus in PG1, and non-casuality and flux are followed by statistically significant values of focus in PG2, YG1, YG2.

We observe the following coincidences of the statistically significant values of $\mathrm{A}, \mathrm{A} \rightarrow \mathrm{B}, \mathrm{B} \rightarrow \mathrm{A}$, non-casuality, flux, and focus in the four groups:

- Evolution of A's choice: PG2 and YG2 between May 16 and 18, 2018;

- A $\rightarrow$ B transitions: PG2 and YG2 between October 6 and 20, 2017;

- B $\rightarrow$ A transitions: PG1 and YG2 between April 8 and May 20, 2016; PG2 and YG1 between March 8 and 15, 2017 and between April 26 and 28, 2017;

- Flux: PG1 and YG2 between April 29 and May 6, 2016, between May 13 and 20, 2016, between June 3 and 10, 2016; PG2 and YG1 between April 26 and 28, 2017; PG2 and YG2 between October 6 and 10, 2017; 


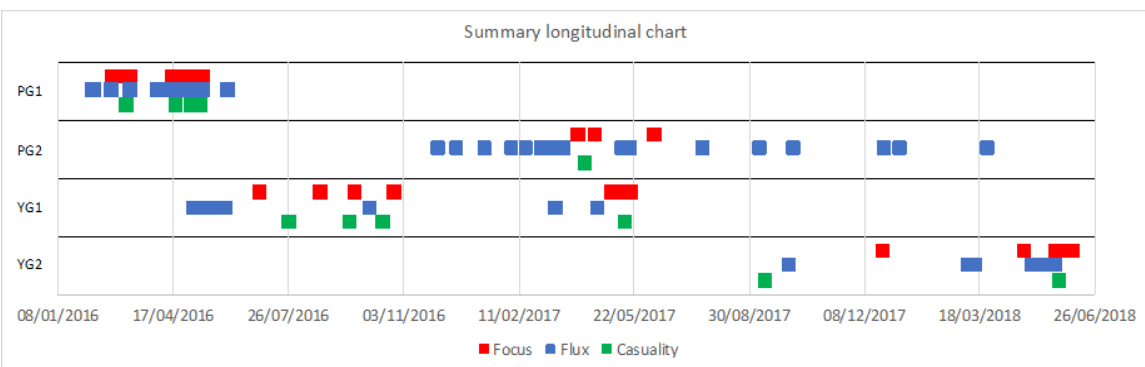

Figure 13. Statistically significant differences between sessions for the percentage of focus $(\mathrm{B} \rightarrow \mathrm{A}-\mathrm{A} \rightarrow \mathrm{B})$, flux $(\mathrm{A} \rightarrow \mathrm{B}+\mathrm{B} \rightarrow \mathrm{A})$, and non-casuality $(\mathrm{A} \rightarrow \mathrm{B}$ vs. $\mathrm{B} \rightarrow \mathrm{A})$ transitions in chronological order for the four groups.

Table 6. Statistically significant probabilities $(p<0.05)$ in the percentage of transitions of choice B $\rightarrow$ A vs. A $\rightarrow$ B for the same session. The cells in red represent statistically significant $(p<0.05)$ differences between the percentages of change in the picture choice $A \rightarrow B$ and $\mathrm{B} \rightarrow \mathrm{A}$, indicating a deviation from a purely statistical fluctuation of the choices.

\begin{tabular}{|c|c|c|c|c|c|c|c|c|c|}
\hline & PG1 & PG2 & YG1 & YG2 & & PG1 & PG2 & YG1 & YG2 \\
\hline S00-01 & 0.82 & 0.98 & 0.64 & 0.01 & S28-29 & & 1.00 & 0.72 & 0.16 \\
\hline S01-02 & 0.15 & 0.52 & 0.59 & 0.22 & S29-30 & & 0.39 & 0.13 & 0.30 \\
\hline S02-03 & 0.20 & 0.21 & 0.41 & 0.41 & S30-31 & & 0.96 & 0.84 & 0.42 \\
\hline S03-04 & 0.01 & 0.84 & 0.81 & 0.93 & S31-32 & & 0.52 & 0.50 & \\
\hline S04-05 & 0.67 & 0.81 & 0.54 & 0.33 & S32-33 & & 0.66 & 0.49 & \\
\hline S05-06 & 1.00 & 0.50 & 0.55 & 0.70 & S33-34 & & 0.18 & 0.88 & \\
\hline S06-07 & 0.80 & 0.89 & 0.34 & 0.57 & S34-35 & & 0.41 & 0.37 & \\
\hline S07-08 & 0.01 & 0.64 & 0.66 & 0.96 & S35-36 & & 0.34 & 0.83 & \\
\hline S08-09 & 0.81 & 0.57 & 0.91 & 0.88 & S36-37 & & 0.87 & 0.99 & \\
\hline S09-10 & 0.00 & 0.56 & 0.68 & 0.10 & S37-38 & & 0.28 & 0.83 & \\
\hline S10-11 & 0.00 & 0.86 & 0.88 & 0.19 & S38-39 & & 1.00 & 0.11 & \\
\hline S11-12 & 0.78 & 0.98 & 0.55 & 0.76 & S39-40 & & 0.54 & 0.25 & \\
\hline S12-13 & 0.56 & 0.56 & 0.48 & 0.90 & S40-41 & & 0.70 & 0.04 & \\
\hline S13-14 & 0.91 & 0.76 & 0.61 & 0.50 & S41-42 & & 0.39 & 0.35 & \\
\hline S14-15 & 0.88 & 0.69 & 0.71 & 0.63 & S42-43 & & 0.44 & 0.97 & \\
\hline S15-16 & & 0.86 & 0.89 & 0.69 & S43-44 & & 0.33 & & \\
\hline S16-17 & & 0.87 & 0.01 & 0.44 & S44-45 & & 0.75 & & \\
\hline S17-18 & & 1.00 & 0.00 & 0.74 & S45-46 & & 0.80 & & \\
\hline S18-19 & & 0.55 & 0.51 & 0.46 & S46-47 & & 0.99 & & \\
\hline S19-20 & & 0.03 & 0.01 & 0.60 & S47-48 & & 0.93 & & \\
\hline S20-21 & & 0.25 & 0.39 & 0.72 & S48-49 & & 0.24 & & \\
\hline S21-22 & & 0.32 & 0.15 & 0.63 & S49-50 & & 0.51 & & \\
\hline S22-23 & & 0.74 & 0.45 & 0.19 & S50-51 & & 0.47 & & \\
\hline S23-24 & & 0.30 & 0.60 & 0.12 & S51-52 & & 0.21 & & \\
\hline S24-25 & & 0.91 & 0.15 & 0.99 & S52-53 & & 0.13 & & \\
\hline S25-26 & & 0.20 & 0.94 & 0.79 & S53-54 & & 0.59 & & \\
\hline S26-27 & & 0.31 & 0.98 & 0.29 & S54-55 & & 0.55 & & \\
\hline
\end{tabular}


A. Fernandez-Rivas et al.

Table 7. Comparison of the flux of choices $\mathrm{A} \rightarrow \mathrm{B}+\mathrm{B} \rightarrow \mathrm{A}$ between successive sessions with the Conover post-hoc test. Two changes of choices are statistically different when $\mathrm{p}<$ 0.05 , and the corresponding cell is red in the table.

\begin{tabular}{|c|c|c|c|c|c|c|c|c|c|}
\hline $\mathrm{A} \rightarrow \mathrm{B}+\mathrm{B} \rightarrow \mathrm{A}$ & PG1 & PG2 & YG1 & YG2 & $\mathrm{A} \rightarrow \mathrm{B}+\mathrm{B} \rightarrow \mathrm{A}$ & PG1 & PG2 & YG1 & YG2 \\
\hline $00 \rightarrow 01-01 \rightarrow 02$ & 0.00 & 0.15 & 0.49 & 0.30 & $27 \rightarrow 28-28 \rightarrow 29$ & & 0.00 & 0.84 & 0.95 \\
\hline $01 \rightarrow 02-02 \rightarrow 03$ & 0.00 & 0.15 & 0.77 & 0.00 & $28 \rightarrow 29-29 \rightarrow 30$ & & 0.00 & 0.79 & 0.09 \\
\hline $02 \rightarrow 03-03 \rightarrow 04$ & 0.30 & 0.92 & 0.87 & 0.13 & $29 \rightarrow 30-30 \rightarrow 31$ & & 0.04 & 0.73 & 0.13 \\
\hline $03 \rightarrow 04-04 \rightarrow 05$ & 0.00 & 1.00 & 0.24 & 0.24 & $30 \rightarrow 31-31 \rightarrow 32$ & & 0.30 & 0.25 & \\
\hline $04 \rightarrow 05-05 \rightarrow 06$ & 0.56 & 0.64 & 0.62 & 0.11 & $31 \rightarrow 32-32 \rightarrow 33$ & & 0.31 & 0.45 & \\
\hline $05 \rightarrow 06-06 \rightarrow 07$ & 0.00 & 0.14 & 0.74 & 0.95 & $32 \rightarrow 33-33 \rightarrow 34$ & & 0.38 & 0.85 & \\
\hline $06 \rightarrow 07-07 \rightarrow 08$ & 0.00 & 0.12 & 0.80 & 0.25 & $33 \rightarrow 34-34 \rightarrow 35$ & & 0.51 & 0.52 & \\
\hline $07 \rightarrow 08-08 \rightarrow 09$ & 0.66 & 0.67 & 0.99 & 0.93 & $34 \rightarrow 35-35 \rightarrow 36$ & & 0.65 & 0.01 & \\
\hline $08 \rightarrow 09-09 \rightarrow 10$ & 0.00 & 0.20 & 0.88 & 0.11 & $35 \rightarrow 36-36 \rightarrow 37$ & & 0.72 & 0.09 & \\
\hline $09 \rightarrow 10-10 \rightarrow 11$ & 0.64 & 0.00 & 0.27 & 0.94 & $36 \rightarrow 37-37 \rightarrow 38$ & & 0.69 & 0.76 & \\
\hline $10 \rightarrow 11-11 \rightarrow 12$ & 0.00 & 0.00 & 0.00 & 0.06 & $37 \rightarrow 38-38 \rightarrow 39$ & & 0.00 & 0.05 & \\
\hline $11 \rightarrow 12-12 \rightarrow 13$ & 0.45 & 0.00 & 0.00 & 0.16 & $38 \rightarrow 39-39 \rightarrow 40$ & & 0.00 & 0.18 & \\
\hline $12 \rightarrow 13-13 \rightarrow 14$ & 0.87 & 0.00 & 0.00 & 0.88 & $39 \rightarrow 40-40 \rightarrow 41$ & & 0.96 & 0.94 & \\
\hline $13 \rightarrow 14-14 \rightarrow 15$ & 0.04 & 0.01 & 0.00 & 0.54 & $40 \rightarrow 41-41 \rightarrow 42$ & & 0.88 & 0.37 & \\
\hline $14 \rightarrow 15-15 \rightarrow 16$ & & 0.52 & 0.00 & 0.68 & $41 \rightarrow 42-42 \rightarrow 43$ & & 0.23 & 0.56 & \\
\hline $15 \rightarrow 16-16 \rightarrow 17$ & & 0.02 & 0.62 & 0.35 & $42 \rightarrow 43-43 \rightarrow 44$ & & 0.13 & & \\
\hline $16 \rightarrow 17-17 \rightarrow 18$ & & 0.00 & 0.14 & 0.20 & $43 \rightarrow 44-44 \rightarrow 45$ & & 0.99 & & \\
\hline $17 \rightarrow 18-18 \rightarrow 19$ & & 0.00 & 0.69 & 0.04 & $44 \rightarrow 45-45 \rightarrow 46$ & & 0.37 & & \\
\hline $18 \rightarrow 19-19 \rightarrow 20$ & & 0.36 & 0.01 & 0.02 & $45 \rightarrow 46-46 \rightarrow 47$ & & 0.47 & & \\
\hline $19 \rightarrow 20-20 \rightarrow 21$ & & 0.07 & 0.28 & 0.29 & $46 \rightarrow 47-47 \rightarrow 48$ & & 0.71 & & \\
\hline $20 \rightarrow 21-21 \rightarrow 22$ & & 0.41 & 0.63 & 0.85 & $47 \rightarrow 48-48 \rightarrow 49$ & & 0.04 & & \\
\hline $21 \rightarrow 22-22 \rightarrow 23$ & & 0.34 & 0.96 & 0.10 & $48 \rightarrow 49-49 \rightarrow 50$ & & 0.69 & & \\
\hline $22 \rightarrow 23-23 \rightarrow 24$ & & 0.00 & 0.62 & 0.51 & $49 \rightarrow 50-50 \rightarrow 51$ & & 0.30 & & \\
\hline $23 \rightarrow 24-24 \rightarrow 25$ & & 0.02 & 0.14 & 0.00 & $50 \rightarrow 51-51 \rightarrow 52$ & & 0.86 & & \\
\hline $24 \rightarrow 25-25 \rightarrow 26$ & & 0.15 & 0.85 & 0.01 & $51 \rightarrow 52-52 \rightarrow 53$ & & 0.55 & & \\
\hline $25 \rightarrow 26-26 \rightarrow 27$ & & 0.93 & 0.88 & 0.01 & $52 \rightarrow 53-53 \rightarrow 54$ & & 0.96 & & \\
\hline $26 \rightarrow 27-27 \rightarrow 28$ & & 0.48 & 0.42 & 0.00 & $53 \rightarrow 54-54 \rightarrow 55$ & & 0.48 & & \\
\hline
\end{tabular}


Table 8. Comparison of the focus of choices $\mathrm{B} \rightarrow \mathrm{A}-\mathrm{A} \rightarrow \mathrm{B}$ between successive sessions with the Conover post-hoc test. Two changes of choices are statistically different when $\mathrm{p}<0.05$, and the corresponding cell is red in the table.

\begin{tabular}{|c|c|c|c|c|c|c|c|c|c|}
\hline $\mathrm{B} \rightarrow \mathrm{A}-\mathrm{A} \rightarrow \mathrm{B}$ & PG1 & PG2 & YG1 & YG2 & $\mathrm{B} \rightarrow \mathrm{A}-\mathrm{A} \rightarrow \mathrm{B}$ & PG1 & PG2 & YG1 & YG2 \\
\hline $00 \rightarrow 01-01 \rightarrow 02$ & 0.19 & 0.63 & 0.42 & 0.09 & $27 \rightarrow 28-28 \rightarrow 29$ & & 0.57 & 0.62 & 0.00 \\
\hline $01 \rightarrow 02-02 \rightarrow 03$ & 0.02 & 0.06 & 0.16 & 0.84 & $28 \rightarrow 29-29 \rightarrow 30$ & & 0.29 & 0.10 & 0.02 \\
\hline $02 \rightarrow 03-03 \rightarrow 04$ & 0.00 & 0.08 & 0.24 & 0.50 & $29 \rightarrow 30-30 \rightarrow 31$ & & 0.23 & 0.25 & 0.10 \\
\hline $03 \rightarrow 04-04 \rightarrow 05$ & 0.01 & 0.42 & 0.92 & 0.39 & $30 \rightarrow 31-31 \rightarrow 32$ & & 0.63 & 0.38 & \\
\hline $04 \rightarrow 05-05 \rightarrow 06$ & 0.70 & 0.84 & 0.80 & 0.70 & $31 \rightarrow 32-32 \rightarrow 33$ & & 0.78 & 0.25 & \\
\hline $05 \rightarrow 06-06 \rightarrow 07$ & 0.74 & 0.48 & 0.21 & 0.95 & $32 \rightarrow 33-33 \rightarrow 34$ & & 0.47 & 0.42 & \\
\hline $06 \rightarrow 07-07 \rightarrow 08$ & 0.02 & 0.85 & 0.24 & 0.69 & $33 \rightarrow 34-34 \rightarrow 35$ & & 0.13 & 0.55 & \\
\hline $07 \rightarrow 08-08 \rightarrow 09$ & 0.02 & 0.44 & 0.64 & 0.68 & $34 \rightarrow 35-35 \rightarrow 36$ & & 0.17 & 0.51 & \\
\hline $08 \rightarrow 09-09 \rightarrow 10$ & 0.00 & 0.84 & 0.73 & 0.23 & $35 \rightarrow 36-36 \rightarrow 37$ & & 0.41 & 0.81 & \\
\hline $09 \rightarrow 10-10 \rightarrow 11$ & 0.00 & 0.42 & 0.79 & 0.01 & $36 \rightarrow 37-37 \rightarrow 38$ & & 0.26 & 0.81 & \\
\hline $10 \rightarrow 11-11 \rightarrow 12$ & 0.00 & 0.86 & 0.63 & 0.36 & $37 \rightarrow 38-38 \rightarrow 39$ & & 0.30 & 0.13 & \\
\hline $11 \rightarrow 12-12 \rightarrow 13$ & 0.70 & 0.61 & 0.28 & 0.80 & $38 \rightarrow 39-39 \rightarrow 40$ & & 0.61 & 0.03 & \\
\hline $12 \rightarrow 13-13 \rightarrow 14$ & 0.45 & 0.46 & 0.26 & 0.50 & $39 \rightarrow 40-40 \rightarrow 41$ & & 0.41 & 0.01 & \\
\hline $13 \rightarrow 14-14 \rightarrow 15$ & 0.69 & 0.59 & 0.98 & 0.65 & $40 \rightarrow 41-41 \rightarrow 42$ & & 0.32 & 0.02 & \\
\hline $14 \rightarrow 15-15 \rightarrow 16$ & & 0.90 & 0.50 & 0.88 & $41 \rightarrow 42-42 \rightarrow 43$ & & 1.00 & 0.47 & \\
\hline $15 \rightarrow 16-16 \rightarrow 17$ & & 1.00 & 0.04 & 0.27 & $42 \rightarrow 43-43 \rightarrow 44$ & & 0.11 & & \\
\hline $16 \rightarrow 17-17 \rightarrow 18$ & & 0.84 & 0.00 & 0.67 & $43 \rightarrow 44-44 \rightarrow 45$ & & 0.43 & & \\
\hline $17 \rightarrow 18-18 \rightarrow 19$ & & 0.54 & 0.00 & 0.74 & $44 \rightarrow 45-45 \rightarrow 46$ & & 0.69 & & \\
\hline $18 \rightarrow 19-19 \rightarrow 20$ & & 0.01 & 0.06 & 0.19 & $45 \rightarrow 46-46 \rightarrow 47$ & & 0.54 & & \\
\hline $19 \rightarrow 20-20 \rightarrow 21$ & & 0.00 & 0.00 & 0.51 & $46 \rightarrow 47-47 \rightarrow 48$ & & 0.81 & & \\
\hline $20 \rightarrow 21-21 \rightarrow 22$ & & 0.97 & 0.33 & 0.70 & $47 \rightarrow 48-48 \rightarrow 49$ & & 0.37 & & \\
\hline $21 \rightarrow 22-22 \rightarrow 23$ & & 0.22 & 0.40 & 0.47 & $48 \rightarrow 49-49 \rightarrow 50$ & & 0.12 & & \\
\hline $22 \rightarrow 23-23 \rightarrow 24$ & & 0.60 & 0.26 & 0.01 & $49 \rightarrow 50-50 \rightarrow 51$ & & 0.25 & & \\
\hline $23 \rightarrow 24-24 \rightarrow 25$ & & 0.50 & 0.08 & 0.17 & $50 \rightarrow 51-51 \rightarrow 52$ & & 0.61 & & \\
\hline $24 \rightarrow 25-25 \rightarrow 26$ & & 0.34 & 0.19 & 0.76 & $51 \rightarrow 52-52 \rightarrow 53$ & & 0.78 & & \\
\hline $25 \rightarrow 26-26 \rightarrow 27$ & & 0.04 & 0.74 & 0.49 & $52 \rightarrow 53-53 \rightarrow 54$ & & 0.38 & & \\
\hline $26 \rightarrow 27-27 \rightarrow 28$ & & 0.14 & 0.31 & 0.01 & $53 \rightarrow 54-54 \rightarrow 55$ & & 0.31 & & \\
\hline
\end{tabular}


We did not find coincidence in statistically significant values for non-casuality and focus.

\section{Discussion}

\subsection{Theoretical Framework}

We believe that, at the moment, the best way to observe the unconscious is to observe its amplification in group dynamics. As it happens in quantum mechanics, the problem of measurement is central also in psychophysics because the unconscious is, by definition, unknowledgeable and not only because it is "unconscious" but also because the "detector" is the cognitive part of the individual, which is deeply influenced by, and indeed built upon, the unconscious.

This analogy relies on the observation that group dynamics, as described by Bion's "basic assumptions," is similar to individual dynamics, in particular in the crucial aspect of the analogy of the mourning process in the individual with the loss of the ideal leader in the group, inspired by the CEdipean constellation.

Considering the presence of a number of potentially connected individuals, we have made the hypothesis that the entanglement effects could be more pronounced in the case of a group setting. We call this "quantum amplification." As said in the Introduction, Jung (Jung \& McGuire, 1925) was aware that the individual psyche is tributary of a layered unconscious, where the layers are the "collective unconscious" of larger and larger groups, such as the family and society.

Jung also speaks of amplification (Jung \& Hull, 1911). For Jung, the "amplification" is the extension and the deepening of a dream-like image by means of associations centered on the dream theme and associations based upon social studies and the history of symbols (mythology, mysticism, folklore, religion, ethnology, art, etc.). Thanks to this, the dream becomes accessible to interpretation (Jung, 1962). If we consider the analogy conveyed by the term "amplification" in the two contexts, unconscious mental processes like dreams can be considered as "microscopic" quantum processes, becoming accessible to conscience only via an amplification and measurement process, in this case, operated by consciousness. This is one more example of the interesting parallels that can be drawn between quantum physics and psychodynamics.

In the case presented in this article, we can suppose that the group situation generated by the DBT skills training is influenced not only by the individual and family situations but also by the environment, such as the large social group.

In quantum physics, a microscopic process is "amplified" by the "observer" to the macroscopic level, and this allows the measurement to take place. It is only after the amplification that a microscopic quantum process can be observed as a physical phenomenon with an act of measurement. The irreversibility of such an act is still an open argument of discussion.

The perspective of this study is to observe if the group dynamics-particularly in a slow-open setting-is also affected by the environment (as in quantum me- 
chanics).

In the present experiment, we noted some differences between parents and adolescents-for example, in the numbers of sessions, similar to what we have observed in one of our previous work of ours (Fernandez-Rivas et al., 2020) but the comparison between parents and adolescents groups will be the subject of a future study.

\subsection{Percentage of A's Answers}

The most striking aspect of our data is that the answers to the questionnaire zero, administered before the training, are very skewed, with values of A's percentages respectively of $75 \%$ for PG1, 70\% for PG2, 64\% for YG1, 65\% for YG2. This is similar to what we found in a previous study of DBT closed groups (Fernandez-Rivas et al., 2020) at the end of the training. We observed a drop to $73 \%$ for PG1, an increase to $80 \%$ for PG2, a reduction to $62 \%$ for YG1, and $56 \%$ for YG2 (Fernandez-Rivas et al., 2020; Trojaola Zapirain et al., 2019).

The trends in the changes of the A's answers are different in the four groups:

- PG1 shows two blocks of significant changes around sessions 3- 6 and 9-12.

- PG2 shows a significant change around 19 - 22, 28 - 29, and 53 - 54.

- YG1 shows a significant change between $17-18,19-20$, and $40-41$.

- YP2 shows significant changes between sessions 0 - 1, 3- 4, 10 - 11, and 26 30.

\subsection{Analysis of "Flux" and "Focus" of the Answers}

The changes $\mathrm{A} \rightarrow \mathrm{B}$ and $\mathrm{B} \rightarrow \mathrm{A}$ in each of the four groups are similar, and this is confirmed that there are few non-casuality significant differences. We recall that we indicate as non-casuality the fact that the changes $\mathrm{A} \rightarrow \mathrm{B}$ and from $\mathrm{B} \rightarrow \mathrm{A}$ are not in equilibrium, i.e., the percentage of changes of the 50 questions are not statistically comparable for the two directions.

The flux, being the sum of $\mathrm{A} \rightarrow \mathrm{B}$ and $\mathrm{B} \rightarrow \mathrm{A}$, present statistically significant values almost at the same moment as $A \rightarrow B$ and $B \rightarrow A$. The statistically significant values for the focus are less than for $\mathrm{A} \rightarrow \mathrm{B}, \mathrm{B} \rightarrow \mathrm{A}$, and flux, apart from the PG1 group, where the significant changes in flux and focus are similar.

The interpretation of this observation is not simple: if we imagine that the originally preferred answer-the A answer of the questionnaire zero-is the "personal" answer independent from the influence of the group, we can suppose that the return to this choice signals a movement "against" the "group continuum" and toward loyalty to the familiar and social clan that we can define as "normal clanity."

The PG1 group, with only 15 sessions, shows a great number of significant variations of the focus between different sessions and a drop of only $2 \%$ in the choice of A from the questionnaire zero to the end of the DBT training (15 sessions). The other groups presented few significant variations of the focus. The variation in the choice of $\mathrm{A}$ from the questionnaire zero to the end of the train- 
ing is different for the four groups. PG1 shows a decrease of 2\% over 15 sessions, PG2 an increase of $20 \%$ over 55 sessions, YG1 a decrease of $6 \%$ over 43 sessions, and YG2 a reduction of $9 \%$ over 31 sessions. Apart from PG1-which ran only for 15 sessions-it is interesting to observe that the number of sessions is inversely proportional to the level of "return" to the original percent of A answers given in the questionnaire zero. This is consistent with the "canonical" group evolution. Towards the end of a group experience, the participants mourn the end of the group and tend to return to their normal clanity to preserve themselves from the group's demise.

In PG2, which has the most sessions, the group continuum seems to have a lesser effect, with an important return to the initial choice of A and few significant values in the non-casuality transitions.

\subsection{Longitudinal Analysis}

Because the groups had a training and therapeutical goal, the fact that some groups took place at the same time was casual and independent from the research objectives. Nevertheless, this overlap in time of the different groups allows a comparison of their evolution in time. Probably because of the slow-open structure of the groups, we observed some coincidence in their evolution which could depend more on external factors than on the group internal evolution.

If we observe the group evolution in calendar time rather than in the number of sessions, we observe the same coincidences in the statistically significant values of some of the parameters considered ( $\mathrm{A}, \mathrm{A} \rightarrow \mathrm{B}, \mathrm{B} \rightarrow \mathrm{A}$, flux), which seem to obey not only the internal evolution of the groups but also other possible external factors. This coincidence was not present for non-casuality and focus.

We underline that if the flux is the expression of changes from $A$ to $B$ plus $B$ to $A$, the non-casuality indicates the statistical difference of the transitions $A \rightarrow B$ and $B \rightarrow A$, and the focus, defined as $B \rightarrow A$ minus $A \rightarrow B$, is the expression of the tendency to revert to the initial choice of pictures before the beginning of the training.

The interaction with the external environment in slow-open groups is probably experienced by the participants in an interactive mode with important clan loyalties: the high "orientation" (A's choices before the constitution of the group) is "recovered" and in a group even exceeded during the sessions and the answers are less casual (less 50\%) than in a state of "group continuum." We note that the participants remain in the group for more or less than $10-12$ sessions. We can suppose that the slow-open group promotes a "clan continuum" due more to the external environment than a group continuum.

\section{Conclusion}

This experiment has been conducted in the years 2017-2019 during Dialectical Behavior Therapy skills training at the Psychiatric Service of Basurto University in Bilbao, Spain. Results of similar experiments have been reported in five pub- 
lications (Fernandez-Rivas et al., 2020; Trojaola-Zapirain et al., 2014, 2015, 2016, 2019). The main conclusion of these studies was that the data were suggesting evidence in favor of the building of a group unconscious following Bion's "basic assumptions," where a strong interaction between the psyches of the group participants is established at the very beginning of the group experience, and then it slowly evolves under the known group dynamics. To describe this phenomenon, Bion has introduced the concept of "valency," indicating the immediacy of the basic assumptions' onset, more analogous to tropisms than to purposive behavior. This effect is enhanced in the group setting by an amplification process whereby groups "amplify emotional reactions, resulting in a combustible process of emotional contagion" (Bion, 1961).

The difficulty of verifying any theory of the unconscious is that we have no way to perform a direct measurement (Atmanspacher, 2004; Cerf \& Adami, 1997, 1998). To circumvent this problem, we have devised an indirect measure based on a questionnaire to be answered by the participants in a group situation.

It is interesting to note that such an experiment is trying to determine whether a psychic situation-the supposed entanglement of the individuals' unconscious in a group situation-has an actual effect on the material world-the answers provided to a questionnaire.

As we said above, the interpersonal relations in the group situation may have a relaxing effect on the neurotic attitudes with a consequent recovery of otherwise non-accessible psychical energy. Otherwise, particularly for adolescents, the feeling of identity loss could bring stress and anxiety and ultimately provoke the exit from the group when the "orientation" (A's choices) is minimal, and the confusion is highest. Psychoanalytically, we would call this a resistance to change. Clan loyalties, common in adolescents when moving from the family-envelope to the friends one may create a strong discomfort and lead to a flight from the group situation. The group continuum is coming from the entanglement of individual psyches toward forming a group unity having its own identity, in this case, a group identity. We can suppose that in slow-open groups, this entanglement is weaker than in closed groups. In slow-open groups, the influence of the external environment seems to be more important, and the conflict between friend clan, family clan, and group clan more manageable.

It might be interesting to attempt to derive some social and practical implications based on the research results, although this is not the primary purpose of the present work. Our study confirms that the slow open setting is the most adapted for the adolescents because it minimizes the adverse effects of the dropouts that are common for this population. While there is a substantial turnover of participants, and the group population oscillates considerably-at least from the perspective of our data-the conflict between clanic loyalties (families and peers, e.g., friends) and the DBT group seems manageable, and the dropouts limited. This could suggest that the slow open setting plays an important role in creating an effective training and therapeutic environment for a population that is demanding but also challenging to treat consistently. 
The participation in the Dialectical Behavior Group Therapy is probably reinforced by the slow-open formula permitting to adolescents and parents a moderate conflict with clanic preexistent loyalties and a more relaxed interaction with group continuum.

\section{Conflicts of Interest}

The authors declare no conflicts of interest regarding the publication of this paper.

\section{References}

Aspect, A., Grangier, P., \& Roger, G. (1982). Experimental Realization of Einstein-Podolsky-Rosen-Bohm Gedankenexperiment. A New Violation of Bell's Inequalities. Physical Review Letters, 49, 91-94. https://doi.org/10.1103/PhysRevLett.49.91

Atmanspacher, H. (2004). Quantum Approaches to Consciousness. In Stanford Encyclopedia of Philosophy. https://plato.stanford.edu/entries/qt-consciousness

Atmanspacher, H., \& Fach, W. (2013). A Structural-Phenomenological Typology of MindMatter Correlations. Journal of Analytical Psychology, 58, 219-244.

https://doi.org/10.1111/1468-5922.12005

Atmanspacher, H., Römer, H., \& Walach, H. (2002). Weak Quantum Theory: Complementarity and Entanglement in Physics and Beyond. Foundations of Physics, 32, 379406. https://doi.org/10.1023/A:1014809312397

Baaquie, B. E., \& Martin, F. (2005). Quantum Psyche-Quantum Field Theory of the Human Psyche. NeuroQuantology, 3, 7-42. https://doi.org/10.14704/nq.2005.3.1.57

Beck, F., \& Eccles, J. (1992). Quantum Aspects of Brain Activity and the Role of Consciousness. Proceedings of the National Academy of Sciences of the United States of America, 89, 11357-11361. https://doi.org/10.1073/pnas.89.23.11357

Bell, J. (1964). On the Einstein Poldolsky Rosen Paradox. Physics, 1, 195-200. https://doi.org/10.1103/PhysicsPhysiqueFizika.1.195

Bell, J. (1966). On the Problem of Hidden Variables in Quantum Mechanics. Reviews of Modern Physics, 38, 447. https://doi.org/10.1103/RevModPhys.38.447

Bion, W. (1961). Experiences in Groups and Other Papers. London: Tavistock Publications Ltd.

Bohr, N. (1935). Can Quantum-Mechanical Description of Physical Reality be Considered Complete? Physical Review, 48, 969-702. https://doi.org/10.1103/PhysRev.48.696

Cerf, N., \& Adami, C. (1997). Quantum Mechanics of Measurement. ArXiv:Quant-Ph/9605002v2.

Cerf, N., \& Adami, C. (1998). What Information Theory Can Tell Us about Quantum Reality. ArXiv:Quant-Ph/9806047v1. arXiv:quant-ph/9806047v1

Conover, W. J. (1999). Practical Nonparametric Statistics (3rd ed.). Hoboken, NJ: Wiley.

Conover, W. J., \& Iman, R., L. (1979). On Multiple-Comparisons Procedures. Tech. Rep. LA-7677-MS, Los Alamos, NM: Los Alamos Scientific Laboratory.

Conte, E., Todarello, O., Federici, A., Vitiello, F., Lopane, M., \& Khrennikov, A. A. (2003). Preliminary Evidence of Quantum like Behavior in Measurement of Mental State. ArXiv:Quant-Ph/0307201v1. arXiv:quant-ph/0307201v1

Einstein, A., Podolsky, B., \& Rosen, N. (1935). Can Quantum-Mechanical Description of Physical Reality Be Considered Complete? Physical Review, 47, 777-780. 
https://doi.org/10.1103/PhysRev.47.777

Fach, W. (2011). Phenomenological Aspects of Complementarity and Entanglement in Exceptional Human Experiences (ExE). Axiomathes, 21, 233-247. https://doi.org/10.1007/s10516-010-9143-7

Fernandez-Rivas, A., Sesma-Pardo, E., Kerexeta, I., Diaz-Cosgaya, A., Vivanco, E., Carminati, F., Torres, M. A. G., Fouassier, C., Martin, F., Demongeot, J., \& Carminati, G. G. (2020). Group Therapy for Adolescents and Parents: Study of Unconscious Orientation. NeuroQuantology, 18, 70-82. https://doi.org/10.14704/nq.2020.18.7.NQ20195

Foulkes, S. H. (1964). Therapeutic Group Analysis. Madison, CT: International Universities Press.

Freeman, W., \& Vitiello, G. (2016). Matter and Mind Are Entangled in Two Streams of Images Guiding Behavior and Informing the Subject Through Awareness. Mind and Matter, 14, 7-24.

Galli Carminati, G., \& Carminati, F. (2006). The Mechanism of Mourning: An Anti-Entropic Mechanism. NeuroQuantology, 4, 186-197. https://doi.org/10.14704/nq.2006.4.2.97

Galli Carminati, G., \& Martin, F. (2008). Quantum Mechanics and the Psyche. Physics of Particles and Nuclei, 39, 560-577. https://doi.org/10.1134/S1063779608040047

Galli Carminati, G., Martin, F., \& Carminati, F. (2017). A Very Simple Quantum Model of Mind and Matter. NeuroQuantology, 15, 186-199.

https://doi.org/10.14704/nq.2017.15.2.1031

Grinberg-Zylberbaum, J., Delaflor, M., Attie, L., \& Goswami, A. (1994). The EinsteinPodolsky-Rosen Paradox in the Brain: The Transferred Potential. Physics Essays, 7, 422.

Hamer, R. M., \& Simpson, P. M. (2009). Last Observation Carried Forward versus Mixed Models in the Analysis of Psychiatric Clinical Trials. American Journal of Psychiatry, 166, 639-641. https://doi.org/10.1176/appi.ajp.2009.09040458

Hameroff, S., \& Penrose, R. (1996). Conscious Events as Orchestrated Spacetime Selections. Journal of Consciousness Studies, 3, 36-53.

Jung, C. G. (1952). Synchronicity: An Acausal Connecting Principle. Princeton, NJ: Princeton University Press. https://doi.org/10.1515/9781400839162

Jung, C. G. (1959). The Archetypes and the Collective Unconscious (2nd ed., Vol. 9). Princeton, NJ: Princeton University Press.

Jung, C. G. (1960). The Structure and Dynamics of the Psyche (Nachdr.). Abingdon-onThames: Routledge.

Jung, C. G. (1962). Memories and Dreams, Reflections. New York: Vintage Editions.

Jung, C. G., \& Hull, R. F. C. (1911). Symbols of Transformation: An Analysis of the Prelude to a Case of Schizophrenia. Princeton, NJ: Princeton University Press.

Jung, C. G., \& McGuire, W. (1925). Analytical Psychology: Notes of the Seminar Given in 1925. Princeton, NJ: Princeton University Press.

https://www.jstor.org/stable/10.2307/j.ctt7rgpf

https://doi.org/10.1515/9781400843077

Jung, C. G., Pauli, W., \& Meyer, C. A. (2001). Atom and Archetype: The Pauli/Jung Letters 1932-1958 (Traduction française: <i>Correspondance 1932-1958</i>, ed. Albin Michel, 2000). Princeton, NJ: Princeton University Press.

Kaës, R. (2010). Le sujet, le lien et le groupe. Groupalité psychique et alliances inconscientes. Cahiers de Psychologie Clinique, 1, 13-40. https://doi.org/10.3917/cpc.034.0013

Khrennikov, A. (2015). Quantum-Like Model of Unconscious-Conscious Dynamics. Fron- 
tiers in Psychology, 6, 997. https://doi.org/10.3389/fpsyg.2015.00997

Linehan, M. (1993). Cognitive-Behavioral Treatment of Borderline Personality Disorder. New York: Guilford Press.

Linehan, M. (2015). DBT Skills Training Manual(2nd ed.). New York: Guilford Press.

Marshall, I. (1989). Consciousness and Bose-Einstein Condensates. New Ideas in Psychology, 7, 73-83. https://doi.org/10.1016/0732-118X(89)90038-X

Martin, F., \& Galli Carminati, G. (2009). Synchronicity, Quantum Mechanics, and Psyche. In H. Atmanspacher, \& H. Primas (Eds.), Recasting Reality: Wolfgang Pauli's Philosophical Ideas and Contemporary Science (pp. 227-243). Berlin: Springer-Verlag. https://doi.org/10.1007/978-3-540-85198-1 11

Martin, F., Carminati, F., \& Galli Carminati, G. (2009). Synchronicity, Quantum Information, and the Psyche. Journal of Cosmology, 3, 580-589.

Martin, F., Carminati, F., \& Galli Carminati, G. (2010). Quantum Information, Oscillations and the Psyche. Physics of Particles and Nuclei, 41, 425-451. https://doi.org/10.1134/S1063779610030032

Martin, F., Carminati, F., \& Galli Carminati, G. (2013). Quantum Information Theory Applied to Unconscious and Consciousness. NeuroQuantology, 11, 16-33. https://doi.org/10.14704/nq.2013.11.1.628

Miller, A. L., Rathus, J. H., \& Linehan, M. (2017). Dialectical Behavior Therapy with Suicidal Adolescents. New York: Guilford Press.

Orlov, Y. F. (1982). The Wave Logic of Consciousness: A Hypothesis. International Journal of Theoretical Physics, 21, 37-53. https://doi.org/10.1007/BF01880263

Penrose, R. (1989). The Emperor's New Mind. Oxford: Oxford University Press. https://doi.org/10.1093/oso/9780198519737.001.0001

Penrose, R. (1994). Shadows of the Mind. Oxford: Oxford University Press.

Pitkänen, M. (1998). Quantum Mind. Quantum Mind Archives, 26. https://journals.sfu.ca/jnonlocality/index.php/jnonlocality/article/download/17/17

Pitkänen, M. (2010). Quantum Mind in TGD Universe. Journal of Nonlocality, 1, 1-42. https://journals.sfu.ca/jnonlocality/index.php/jnonlocality/article/download/17/17

R Core Team (2020). R: A Language and Environment for Statistical Computing. R Foundation for Statistical Computing. https://www.R-project.org/

Richens, J. F., Selby, J. H., \& Al-Safi, S. W. (2017). Entanglement Is Necessary for Emergent Classicality in All Physical Theories. Physical Review Letters, 119, Article ID: 080503. https://doi.org/10.1103/PhysRevLett.119.080503

Sabbadini, S., \& Vitiello, G. (2019). Entanglement and Phase-Mediated Correlations in Quantum Field Theory. Application to Brain-Mind States. Applied Sciences, 9, 3203. https://doi.org/10.3390/app9153203

Schrödinger, E. (1935). Discussion of Probability Relations between Separated Systems. Mathematical Proceedings of the Cambridge Philosophical Society, 31, 555-563. https://doi.org/10.1017/S0305004100013554

Schrödinger, E. (1936). Probability Relations between Separated Systems. Mathematical Proceedings of the Cambridge Philosophical Society, 32, 446-452.

https://doi.org/10.1017/S0305004100019137

Trojaola-Zapirain, B., Carminati, F., Fernandez Rivas, M. A., Gonzalez Torres, A., Gonzales de Mendivil, E., Fouassier, C., Martin, F., Demongeot, J., \& Galli Carminati, G. (2019). An Update and Generalization of Group Unconscious Orientation in OMIE Group Training for Therapists. Neuroquantology, 17, 14-30. 
https://doi.org/10.14704/nq.2019.17.1.1918

Trojaola-Zapirain, B., Carminati, F., Gonzalez Torres, A., Gonzales de Mendivil, E., Fouassier, C., Gex-Fabry, M., Martin, F., Labarere, J., Demongeot, J., Lorincz, E. N., \& Galli Carminati, G. (2014). Group Unconscious Common Orientation: Exploratory Study at the Basque Foundation for the Investigation of Mental Health Group Training for Therapists. Neuroquantology, 12, 139-150.

https://doi.org/10.14704/nq.2014.12.1.709

Trojaola-Zapirain, B., Carminati, F., Gonzalez Torres, A., Gonzales de Mendivil, E., Fouassier, C., Gex-Fabry, M., Martin, F., Labarere, J., Demongeot, J., Lorincz, E. N., \& Galli Carminati, G. (2015). Addendum on Entropy to the Exploratory Study on Group Unconscious at the Basque Foundation for the Investigation of Mental Health Group Training for Therapists. Neuroquantology, 13, 49-56.

https://doi.org/10.14704/nq.2015.13.1.780

Trojaola-Zapirain, B., Carminati, F., Gonzalez Torres, A., Gonzales de Mendivil, E., Fouassier, C., Gex-Fabry, M., Martin, F., Labarere, J., Demongeot, J., Lorincz, E. N., \& Galli Carminati, G. (2016). A Comparison of the Evolution and Entropy of Responses to Picture Choices on an "Absurdum Questionnaire" between Members of Two Different Training Groups. Neuroquantology, 14, 501-513.

https://doi.org/10.14704/nq.2016.14.3.919

Vergopoulos, T. (1983). La sensibilisation à la dynamique de groupe d'aprés W. R. Bion et S.H. Foulkes. Médecine et Hygiène, 1, 3149-3155.

Vitiello, G. (2003). Quantum Dissipation and Information: A Route to Consciousness Modelling. NeuroQuantology, 1, 266-279. https://doi.org/10.14704/nq.2003.1.2.15

Walach, H., \& Römer, H. (2011). Generalized Entanglement-A Nonreductive Option for a Phenomenologically Dualist and Ontologically Monist View of Consciousness. In $\mathrm{H}$. Walach, S. Schmidt, \& W. B. Jonas (Eds.), Neuroscience, Consciousness and Spirituality (Vol. 1, pp. 81-95). Dordrecht: Springer.

https://doi.org/10.1007/978-94-007-2079-4 6

Zanello, A., Rouget-Weber, B., Gex Fabry, M. G., Maercker, A., \& Guimon, J. (2004). New Instrument to Assess Social Functioning in Mental Health Settings. European Journal of Psychiatry, 18, 76-78.

Zurek, H. (1981). Pointer Basis of Quantum Apparatus: Into What Mixture Does the Wave Packet Collapse? Physical Review D, 24, 1516.

https://doi.org/10.1103/PhysRevD.24.1516 